\title{
Equilibria of channel selection games in parallel multiple access channels
}

\author{
Samir M Perlaza ${ }^{1 *}$, Samson Lasaulce ${ }^{2}$ and Mérouane Debbah ${ }^{3}$
}

\begin{abstract}
In this article, the parallel multiple access channel is studied under the assumption that transmitters maximize their individual spectral efficiency by selfishly tuning their power allocation policy. Two particular scenarios are studied: (a) transmitters are allowed to use all the available channels; and (b) transmitters are constrained to use a single channel. Both scenarios are modeled by one-shot games and the corresponding sets of Nash equilibria (NE) are fully characterized under the assumption that the receiver treads the multiple access interference as noise. In both cases, the set of NE is non-empty. In the case in which transmitters use a single channel, an upper bound of the cardinality of the NE set is provided in terms of the number of transmitters and number of channels. In particular, it is shown that in fully loaded networks, the sum spectral efficiency at the NE in scenario (a) is at most equal to the sum spectral efficiency at the NE in scenario (b). A formal proof of this observation, known in general as a Braess paradox, is provided in the case of two transmitters and two channels. In general scenarios, we conjecture that the same effect holds as long as the network is kept fully loaded, as shown by numerical examples. Moreover, the price of anarchy and the price of stability in both games are also studied. Interestingly, under certain conditions on the channel gains, Pareto optimality can be achieved at some NE if and only if the number of channels equals or exceeds the number of transmitters. Finally, simulations are presented to verify the theoretical results.
\end{abstract}

\section{Introduction}

Multiple access channels (MAC) correspond to a communication scenario where several transmitters communicate with a single receiver through a common channel [1]. In parallel MAC, each transmitter can exploit a common set of orthogonal channels to communicate with the receiver. Often, channel orthogonality is assumed in the frequency domain, and thus, channels can be understood as different non-overlapping frequency bands. This model allows one to study communication scenarios such as 802.11-based wireless local area networks [2,3], distributed soft or hard handovers in cellular systems [4], or throughput-maximizing power control in multi-carrier code division multiple access (MC-CDMA) systems [5].

In this article, we analyze the parallel MAC assuming that transmitters selfishly maximize their individual spectral efficiency (ISE) by autonomously selecting a single

\footnotetext{
${ }^{*}$ Correspondence: perlaza@princeton.edu

1 Department of Electrical Engineering, Princeton University, Equad, 19 Olden

Street, Princeton, NJ 08544, USA

Full list of author information is available at the end of the article
}

channel to perform their transmission. Here, the channel selection (CS) policy is not imposed by the receiver to the transmitters, which justifies the terms decentralized parallel MAC. The motivation for studying this scenario and, in particular, the limitation of using a single channel for transmitting stems from the fact that this is often a practical constraint in some wireless networks, for instance, Wi-Fi networks. Moreover, the choice of enforcing radio devices to use only a subset of all the available channels has been proved to be beneficial in the case of rate-efficient centralized parallel MAC when using successive interference cancellation [4]. In the case of energy-efficient decentralized parallel MAC, it has been shown that using a single channel is a dominant strategy [5]. Within this framework, we study this scenario, to which we refer to as CS problem, using a one-shot game model. The players (the transmitters) have discrete action sets (the channels) and their utility function (performance metric) corresponds to their ISE. Our interest focuses on the analysis of the set of Nash equilibria (NE) [6] of this game. The relevance of the NE relies, in part, on the fact that it describes a network state where the channel used by each transmitter is individually optimal with respect to

\section{Springer}

(c) 2013 Perlaza et al: licensee Springer. This is an Open Access article distributed under the terms of the Creative Commons Attribution License (http://creativecommons.org/licenses/by/2.0), which permits unrestricted use, distribution, and reproduction in any medium, provided the original work is properly cited. 
the channels adopted by all the other transmitters in the network. Another reason is that an NE can be reached in a fully decentralized fashion when radio devices interact during certain time following particular behavioral rules $[7,8]$.

We distinguish the CS game described above from the power allocation (PA) game. In the PA game, transmitters can simultaneously use all the available channels, and thus, the set of actions is a convex and closed set [9]. Indeed, the PA problem in decentralized parallel MAC has been well investigated in the wireless literature [9-13]. In these works, the main contribution consists in conducting a complete analysis of the set of NE of the corresponding one-shot games. Nonetheless, very few is known about the set of equilibria in decentralized parallel MAC, with CS policies. As we shall see, by dropping the convexity of the set of actions, fundamental differences arise. For instance, the uniqueness of the NE no longer holds.

Although the parallel MAC is, in terms of signal model, a special case of the multiple-input multipleoutput (MIMO) interference channel (IC), the NE analysis of the MIMO-IC [14-18] does not directly apply to the case of parallel MAC. First, [10,11] address the case of fast fading links and shows substantial differences between both models especially in terms of the uniqueness of the NE. Second, even if identical channel variation models are considered in the MIMO-IC and parallel MAC, the sufficient condition for the uniqueness of the NE derived in $[14,19]$ for static or block fading IC can be shown to hold with probability zero in parallel MAC [9]. In particular, the sufficient condition for the uniqueness of the NE provided in [14] is not necessary in general [20]. The (more explicit) sufficient conditions for uniqueness given by Scutari et al. [19] are generally not verified in parallel MAC. More precisely, from [19] it is implied that there exists a unique pure NE with high probability when for each point-to-point communication the signal dominates the interference. This condition is clearly not verified in parallel MAC. For instance, in a 2-user parallel MAC, if one user's signal is dominated by the interference, the converse holds for the other user. Finally, we highlight that in the parallel MAC, when single-user decoding (SUD) is assumed at the receiver, both the compact PA and CS games have a special structure, namely, they are potential games [21] or more specifically, best-response potential games [22]. This structure is not observed in the case of the MIMO-IC model. In Section 3, we discuss the implications and advantages derived from this fact.

Within this context, the main contributions of this article are described hereunder.

- The set of NE of the decentralized parallel MAC is fully described in the case of CS policies and SUD at the receiver. This set is shown to be non-empty and an upper bound of its cardinality is provided as a function of the number of transmitters and available channels.

- In the 2-transmitter 2-channel case, it is formally proved that for any realization of the channel gains, there always exists at least one NE in the CS game that produces a higher or equal network spectral efficiency (NSE) than the unique NE in the PA game. In wireless communications, this kind of observations is often associated with a Braess-type paradox [23] as in $[20,24,25]$, where similar observations have been made in other scenarios. For an arbitrary number of transmitters and channels, we only provide numerical results that support the aforementioned claim.

- The set of NE of the decentralized parallel MAC is also studied in the asymptotic regime, that is for a large number of transmitters in the case of CS policies. In this context, we provide closed-form expressions of the fraction of players which transmit over each channel as a function of the ratio between the channel bandwidth and the total available bandwidth (sum of all channel bandwidths).

- Finally, we study the efficiency of the set of equilibria in terms of the price of anarchy (PoA) and the price of stability (PoS). We provide closed-form conditions over which no loss of performance is observed at the equilibrium in both the PA and the CS games.

The content of this article can briefly be summarized as follows. In Section 2, the system and game models are described. In Section 3, we revisit the existing results regarding the PA problem and we provide new results on the CS problem in terms of existence and uniqueness of the NE. In Section 4, the contribution aforementioned are fully detailed. In Section 5, we present simulation results in order to verify our theoretical results. Finally, the article is concluded by Section 6 .

\section{Models}

\subsection{System model}

Let us define the sets $\mathcal{K} \triangleq\{1, \ldots, K\}$ and $\mathcal{S} \triangleq\{1, \ldots, S\}$. Consider a parallel MAC with $K$ transmitters and $S$ channels (namely non-overlapping bands). Denote by $\boldsymbol{y}=$ $\left(y_{1}, \ldots, y_{S}\right)^{T}$ the $S$-dimensional vector representing the received signal, which can be written in the baseband at the symbol rate as follows

$$
\boldsymbol{y}=\sum_{k=1}^{K} \boldsymbol{H}_{k} \boldsymbol{x}_{k}+\boldsymbol{w} .
$$

Here, $\forall k \in \mathcal{K}, \boldsymbol{H}_{k}$ is the channel transfer matrix from transmitter $k$ to the receiver, $\boldsymbol{x}_{k}$ is the vector of symbols transmitted by transmitter $k$, and vector $\boldsymbol{w}$ represents 
the noise observed at the receiver. We will exclusively deal with the scenario where the matrices $\boldsymbol{H}_{k}$ are $\boldsymbol{S}$ dimensional diagonal matrices (parallel MAC), i.e., $\boldsymbol{H}_{k}=$ $\operatorname{diag}\left(h_{k, 1}, \ldots, h_{k, S}\right)$. In our analysis, block fading channels are assumed. Hence, for each channel use, the entries $h_{k, s}$, for all $(k, s) \in \mathcal{K} \times \mathcal{S}$, are time-invariant realizations of a complex circularly symmetric Gaussian random variable with zero mean and unit variance. Here, we assume that each transmitter is able to perfectly estimate its own channel realizations (coherent communications), i.e., the channels $h_{k, 1} \ldots h_{k, S}$ for transmitter $k$. The vector of transmitted symbols $\boldsymbol{x}_{k}, \forall k \in \mathcal{K}$ is an $S$-dimensional complex circularly symmetric Gaussian random variable with zero mean and covariance matrix $\boldsymbol{P}_{k}=\mathbb{E}\left(\boldsymbol{x}_{k} \boldsymbol{x}_{k}^{H}\right)=$ $\operatorname{diag}\left(p_{k, 1}, \ldots, p_{k, S}\right)$. Assuming the transmit symbols to be Gaussian and independent is optimal in terms of spectral efficiency, as shown in [26,27]. For all $(k, s) \in \mathcal{K} \times \mathcal{S}$, $p_{k, s}$ represents the transmit power allocated by transmitter $k$ over channel $s$ and transmitters are power-limited, that is

$$
\forall k \in \mathcal{K}, \quad \sum_{s=1}^{S} p_{k, s} \leqslant p_{k, \max }
$$

where $p_{k, \max }$ is the maximum transmit power of transmitter $k$. A PA vector for transmitter $k$ is any vector $\boldsymbol{p}_{k}=\left(p_{k, 1}, \ldots, p_{k, S}\right)$ with non-negative entries satisfying (2). The noise vector $\boldsymbol{w}$ is an $S$-dimensional zero mean Gaussian random variable with independent, equal variance real and imaginary parts. Here, $\mathbb{E}\left(\boldsymbol{w} \boldsymbol{w}^{H}\right)=$ $\operatorname{diag}\left(\sigma_{1}^{2}, \ldots, \sigma_{S}^{2}\right)$, where $\sigma_{s}^{2}$ represents the noise power over channel $s$. We, respectively, denote the noise spectral density and the bandwidth of channel $s$ by $N_{0}$ and $B_{s}$, thus, $\sigma_{s}^{2}=N_{0} B_{s}$. The total bandwidth is denoted by $B=\sum_{s=1}^{S} B_{s}$.

\subsection{Game models}

The PA and CS problems described in Section 1 can, respectively, be modeled by the following two noncooperative static games in strategic form (with $i \in\{a, b\}$ ):

$$
\mathcal{G}_{(i)}=\left(\mathcal{K},\left(\mathcal{P}_{k}^{(i)}\right)_{k \in \mathcal{K}},\left(u_{k}\right)_{k \in \mathcal{K}}\right)
$$

In both games, the set of transmitters $\mathcal{K}$ is the set of players. An action of a given transmitter $k \in \mathcal{K}$ is a particular PA scheme, i.e., an $S$-dimensional PA vector $\boldsymbol{p}_{k}=$ $\left(p_{k, 1}, \ldots, p_{k, S}\right) \in \mathcal{P}_{k}^{(i)}$, where $\mathcal{P}_{k}^{(i)}$ is the set of all possible PA vectors which transmitter $k$ can use either in the game $\mathcal{G}_{(a)}(i=a)$ or in the game $\mathcal{G}_{(b)}(i=b)$. An action profile of the game $i \in\{a, b\}$ is a super vector

$$
\boldsymbol{p}=\left(\boldsymbol{p}_{1}, \ldots, \boldsymbol{p}_{K}\right) \in \mathcal{P}^{(i)},
$$

where $\mathcal{P}^{(i)}$ is a set obtained from the Cartesian product of all the action sets, i.e., $\mathcal{P}^{(i)}=\mathcal{P}_{1}^{(i)} \times \cdots \times \mathcal{P}_{K}^{(i)}$, where

$$
\begin{gathered}
\mathcal{P}_{k}^{(a)}=\left\{\left(p_{k, 1}, \ldots, p_{k, S}\right) \in \mathbb{R}^{S}: \forall s \in \mathcal{S}, p_{k, s} \geqslant 0,\right. \\
\left.\sum_{s \in \mathcal{S}} p_{k, s} \leqslant p_{k, \max }\right\}, \text { and } \\
\mathcal{P}_{k}^{(b)}=\left\{p_{k, \text { max }} \boldsymbol{e}_{s}: \forall s \in \mathcal{S}, \boldsymbol{e}_{s}=\left(e_{s, 1}, \ldots, e_{s, S}\right),\right. \\
\left.\forall r \in \mathcal{S} \backslash s, e_{s, r}=0, \text { and } e_{s, s}=1\right\} .
\end{gathered}
$$

In the sequel, we respectively refer to the games $\mathcal{G}_{(a)}$ and $\mathcal{G}_{(b)}$ as the PA game and CS game. Moreover, we drop the super-indices ${ }^{(a)}$ or ${ }^{(b)}$ when interchangeably referring to the game $\mathcal{G}_{(a)}$ or $\mathcal{G}_{(b)}$.

Let us denote by $\boldsymbol{p}_{-k}$ any vector in the set

$$
\mathcal{P}_{-k}^{(i)} \triangleq \mathcal{P}_{1}^{(i)} \times \cdots \times \mathcal{P}_{k-1}^{(i)} \times \mathcal{P}_{k+1}^{(i)} \times \cdots \times \mathcal{P}_{K}^{(i)}
$$

with $(i, k) \in\{a, b\} \times \mathcal{K}$. For a given $k \in \mathcal{K}$, the vector denoted by $\boldsymbol{p}_{-k}$ represents the strategies adopted by all the players other than player $k$. With a slight abuse of notation, we can write any vector $\boldsymbol{p} \in \mathcal{P}^{(i)}$ as $\left(\boldsymbol{p}_{k}, \boldsymbol{p}_{-k}\right)$, in order to emphasize the $k$ th component of the super vector $\boldsymbol{p}$. The utility for player $k$ in the game $\mathcal{G}_{(i)}$ is its spectral efficiency $u_{k}: \mathcal{P}^{(i)} \rightarrow \mathbb{R}_{+}$, and

$$
u_{k}\left(\boldsymbol{p}_{k}, \boldsymbol{p}_{-k}\right)=\sum_{s \in \mathcal{S}} \frac{B_{s}}{B} \log _{2}\left(1+\gamma_{k, s}\right)[\mathrm{bps} / \mathrm{Hz}],
$$

where $\gamma_{k, s}$ is the signal-to-interference plus noise ratio (SINR) seen by player $k$ over its channel $s$, i.e.,

$$
\gamma_{k, s}=\frac{p_{k, s} g_{k, s}}{\sigma_{s}^{2}+\sum_{j \in \mathcal{K} \backslash\{k\}} p_{j, s} g_{j, s}},
$$

and $g_{k, s} \triangleq\left|h_{k, s}\right|^{2}$. Note that from (6), it is implied that SUD is used at the receiver. The choice of SUD is basically due to scalability (in terms of signaling cost) and fairness for the decoding scheme. Clearly, optimality is not sought here, nonetheless, these constraints are inherit to the decentralized nature of the network.

As a solution concept for both $\mathcal{G}_{(a)}$ and $\mathcal{G}_{(b)}$, we focus on the notion of NE [6], which we define, using our notation, as follows

Definition 1 (Pure NE). In the non-cooperative games in strategic form $\mathcal{G}_{(i)}$, with $i \in\{a, b\}$, an action profile $\boldsymbol{p} \in$ $\mathcal{P}^{(i)}$ is a pure NE if it satisfies, for all $k \in \mathcal{K}$ and for all $\boldsymbol{p}_{k}^{\prime} \in \mathcal{P}_{k}^{(i)}$, that

$$
u_{k}\left(\boldsymbol{p}_{k}, \boldsymbol{p}_{-k}\right) \geqslant u_{k}\left(\boldsymbol{p}_{k}^{\prime}, \boldsymbol{p}_{-k}\right) \text {. }
$$

The relevance of the NE is that at such state, the PA or CS policy chosen by any transmitter is optimal with respect to the choice of all the other transmitters. Thus, in a decentralized network, the NE is a stable network state, 
since no player has a particular interest in deviating from the actual state.

In the following, we provide some fundamental results which we use in the further analysis of the games $\mathcal{G}_{(a)}$ and $\mathcal{G}_{(b)}$.

\section{Auxiliary results}

In this section, we introduce some existing results on the existence and uniqueness of the NE in the games $\mathcal{G}_{(a)}$ and $\mathcal{G}_{(b)}$. For doing so, we use the fact that both games $\mathcal{G}_{(a)}$ and $\mathcal{G}_{(b)}$ have been shown to be potential games (PG) $[21,28]$ in $[4,29]$, respectively. We conclude this section by introducing a new result which allows us to establish an upper bound on the number of NE that the game $\mathcal{G}_{(b)}$ might possess.

\subsection{General results}

The analysis presented in the following strongly relies on the fact that both games $\mathcal{G}_{(a)}$ and $\mathcal{G}_{(b)}$ are potential games. Thus, for the sake of completeness, we define exact PGs using our notation.

Definition 2 (Exact PG). Any game in strategic form defined by the triplet

$$
\mathcal{G}=\left(\mathcal{K},\left(\mathcal{P}_{k}\right)_{k \in \mathcal{K}},\left(u_{k}\right)_{k \in \mathcal{K}}\right)
$$

is an exact potential game if there exists a function $\phi(\boldsymbol{p})$ for all $\boldsymbol{p} \in \mathcal{P}=\mathcal{P}_{1} \times \cdots \times \mathcal{P}_{K}$ such that for all players $k \in \mathcal{K}$ and for all $\boldsymbol{p}_{k}^{\prime} \in \mathcal{P}_{k}$, it holds that

$$
u_{k}\left(\boldsymbol{p}_{k}, \boldsymbol{p}_{-k}\right)-u_{k}\left(\boldsymbol{p}_{k}^{\prime}, \boldsymbol{p}_{-k}\right)=\phi\left(\boldsymbol{p}_{k}, \boldsymbol{p}_{-k}\right)-\phi\left(\boldsymbol{p}_{k}^{\prime}, \boldsymbol{p}_{-k}\right)
$$

Note that the utility function of player $k$ can be written as follows,

$$
u_{k}\left(\boldsymbol{p}_{k}, \boldsymbol{p}_{-k}\right)=\phi(\boldsymbol{p})-v_{k}\left(\boldsymbol{p}_{-k}\right)
$$

where

$$
\begin{aligned}
\phi(\boldsymbol{p}) & =\sum_{s \in \mathcal{S}} \frac{B_{s}}{B} \log _{2}\left(\sigma_{s}^{2}+\sum_{k=1}^{K} p_{k, s} g_{k, s}\right) \text { and } \\
v_{k}\left(\boldsymbol{p}_{-k}\right) & =\sum_{s \in \mathcal{S}} \frac{B_{s}}{B} \log _{2}\left(\sigma_{s}^{2}+\sum_{j \in \mathcal{K} \backslash\{k\}} p_{j, s} g_{j, s}\right) .
\end{aligned}
$$

Interestingly, the first term $\phi(\boldsymbol{p})$ does not depend on the index of $k$ and the term $v_{k}\left(\boldsymbol{p}_{-k}\right)$ does not depend on the actions of player $k$. Thus, the results initially mentioned by the authors of $[4,29]$ follow immediately from (8) and Definition 2.
Lemma 1. The strategic form games $\mathcal{G}_{(i)}$, with $i \in\{a, b\}$, are exact potential games with potential function $\phi(\boldsymbol{p})$ : $\mathcal{P}^{(i)} \rightarrow \mathbb{R}_{+}$defined in (9).

The relevance of PG relies on the fact that it is a class of games for which the existence of at least one pure NE is always guaranteed [21]. In addition, many known learning procedures, such as, best response dynamics, fictitious play, and some reinforcement learning dynamics converge in PG. As a consequence, any of these dynamics can be used to implement algorithms to achieve an equilibrium in a fully decentralized fashion. Nonetheless, we leave the design of decentralized techniques for achieving NE out of the scope of this article and we focus on the analysis of the equilibria. We refer the interested readers to $[7,8,13,14,30]$ for more details. In the following, we use Lemma 1 to analyze the set of NE of both our PA game $\mathcal{G}_{(a)}$ and our CS game $\mathcal{G}_{(b)}$.

\subsection{Known results concerning the PA game $\mathcal{G}_{(a)}$}

In the following, we comment on the existence and uniqueness of the NE in $\mathcal{G}_{(a)}$.

\subsubsection{Existence of an NE}

Regarding the existence of pure NE, the following lemma is an immediate consequence of our Lemma 1 and Lemma 4.3 in [21].

Lemma 2 (Existence of a pure NE). The game $\mathcal{G}_{(\text {a })}$ has always at least one NE in pure strategies.

Regarding the existence of a mixed NE (i.e., a probability distribution on the possible actions which verifies Definition 1), it follows from [31], that the existence of at least one NE in mixed strategies always exists. This is basically because the action spaces $\mathcal{P}_{k}^{(a)}$ are compact spaces and the utility functions are continuous with respect to the action profile. However, in compact strategy spaces, mixed strategies are generally less attractive due to the difficulty of its implementation in wireless communications systems [8].

\subsubsection{Uniqueness of the pure NE}

In the game $\mathcal{G}_{(a)}$, the uniqueness of the NE has been shown to hold with probability one [9].

Theorem 1 (NE uniqueness in parallel MAC). The game $\mathcal{G}_{(a)}$ has almost surely a unique pure $N E$.

A formal proof of Theorem 1 is provided in [9]. This proof is based on the concept of degeneracy which allows one to characterize the directions along which the potential remains constant. A simpler proof for the special case of 2-players and 2-channels is given in [32]. 


\subsubsection{Determination of the NE}

From Definition 2, it follows that the unique NE in pure strategies, denoted by $\boldsymbol{p}^{\dagger}$, is the unique solution of the following optimization problem:

$$
\arg \max _{p \in \mathcal{P}^{(a)}} \phi(\boldsymbol{p}) .
$$

The components of the PA vector $\boldsymbol{p}^{\dagger}$ in (11) are for all $(k, s) \in \mathcal{K} \times \mathcal{S}$,

$$
p_{k, s}^{\dagger}=\left[\frac{B_{s}}{B} \frac{1}{\beta_{k}}-\frac{\sigma_{s}^{2}+\sum_{j \in \mathcal{K} \backslash\{k\}} p_{j, s}^{\dagger} g_{j, s}}{g_{k, s}}\right]^{+},
$$

where $\beta_{k}$ is a Lagrangian multiplier chosen to saturate the power constraints (2). Note that this result shows the connections between the notion of NE and the well-known water-filling PA policy [13]. For a further discussion on this connection, the reader is referred to $[7,8]$.

\subsection{New results concerning the CS game $\mathcal{G}_{(b)}$}

In the game $\mathcal{G}_{(b)}$, it can be checked that given a vector $\boldsymbol{p}_{-k} \in \mathcal{P}_{-k}^{(b)}$, it follows that $\forall k \in \mathcal{K}$ and $\forall p_{k} \in\left[0, p_{k, \max }\right]$, the potential function satisfies that $\phi\left(p_{k} \boldsymbol{e}_{s}, \boldsymbol{p}_{-k}\right) \leqslant$ $\phi\left(p_{k, \max } \boldsymbol{e}_{s}, \boldsymbol{p}_{-k}\right)$, where $\boldsymbol{e}_{s}=\left(e_{s, 1}, \ldots, e_{s, S}\right) \in \mathbb{R}^{S}, \forall r \in$ $\mathcal{S} \backslash s, e_{s, r}=0$, and $e_{s, s}=1$. Thus, the problem of transmit power control disappears and there is no lost of generality by choosing the action sets as $\mathcal{P}_{k}^{(b)}$. Technically, the main difference between $\mathcal{G}_{(a)}$ and $\mathcal{G}_{(b)}$ is that the latter is a finite game $(|\mathcal{K} \times \mathcal{S}|<+\infty)$. In the following, we investigate the consequences of this fact on the existence and multiplicity of the NE.

\subsubsection{Existence of a pure NE}

Regarding the existence of at least one NE in pure strategies, we have that from our Lemma 1 and Corollary 2.2 of [21], the following lemma holds.

Lemma 3 (Existence of a pure NE). The game $\mathcal{G}_{(b)}$ has always at least one NE in pure strategies.

Regarding the existence of an equilibrium in mixed strategies, we have that given that the actions sets are discrete and finite, then the existence of at least one NE in mixed strategies is ensured [6].

\subsubsection{Multiplicity of the pure NE}

In the following, we show that multiple NE might exists in the game $\mathcal{G}_{(b)}$.

Proposition 1. Let $\hat{K} \in \mathbb{N}$ be the highest even number which is less or equal to $K$. Then, the game $\mathcal{G}_{(b)}$ has L pure NE strategy profiles, where,

$$
1 \leqslant L \leqslant 1+(S-1) \sum_{i \in\{2,4, \ldots, \hat{K}\}}\left(\begin{array}{c}
K \\
i
\end{array}\right) .
$$

The proof of Proposition 1 is given in Appendix. This proof relies on the fact that whenever a player deviates from a given action profile $\boldsymbol{p}$, it achieves a different utility. That is, given a set of channels $\left\{g_{i j}\right\}_{\forall(i, j) \in \mathcal{K} \times \mathcal{S}}$, it holds that $u_{k}(\boldsymbol{p}) \neq u_{k}\left(\boldsymbol{p}^{\prime}\right)$, where $\boldsymbol{p}^{\prime}$ is obtained by letting only one transmitter to deviate from $p$. This holds from the fact that the channel realizations are drawn from a continuous distribution and thus, $\operatorname{Pr}\left(u_{k}(\boldsymbol{p})=u_{k}\left(\boldsymbol{p}^{\prime}\right) \mid \boldsymbol{p} \neq \boldsymbol{p}^{\prime}\right)=0$. Thus, under this condition, if $\boldsymbol{p}$ is an NE, then $\boldsymbol{p}^{\prime}$ is not and vice versa. Following this procedure, a set of action profiles can be eliminated and only some actions profiles which are NE candidates are left over. In this way, a bound on the maximum number of equilibria can be provided independently of the exact channel realizations and only relying on the parameters $K$ and $S$. Indeed, this is one of the main properties of the bound provided by Proposition 1 since, a calculation of the exact number of $\mathrm{NE}$ becomes cumbersome when the number of transmitters $K$ and channels $S$ grows to infinity. As we shall see, the bound in Proposition 1 holds with strict equality in the case of $K=2$ transmitters and $S=2$ channels, that is, $1 \leqslant L \leqslant 2$. For an arbitrary number of transmitters $K>2$ and channels $S>2$, the bound is not tight. This is basically because some of the action profiles obtained using the elimination processes described before might mutually be exclusive of the set of NE. For instance, in the game $\mathcal{G}_{(b)}$ with $K=3$ and $S=2$, the set of PA vectors $p_{\max }\left(\boldsymbol{e}_{1}, \boldsymbol{e}_{1}, \boldsymbol{e}_{1}\right), p_{\max }\left(\boldsymbol{e}_{1}, \boldsymbol{e}_{2}, \boldsymbol{e}_{2}\right), p_{\max }\left(\boldsymbol{e}_{2}, \boldsymbol{e}_{2}, \boldsymbol{e}_{1}\right)$, and $p_{\max }\left(\boldsymbol{e}_{2}, \boldsymbol{e}_{1}, \boldsymbol{e}_{2}\right)$ might all be NE candidates. Nonetheless, if $p_{\max }\left(\boldsymbol{e}_{1}, \boldsymbol{e}_{1}, \boldsymbol{e}_{1}\right)$ is an equilibrium for a given vector of channels $\left\{g_{i j}\right\}_{\forall(i, j) \in \mathcal{K} \times \mathcal{S}}$, then the other three action sets are not NE for the same set of channels and vice versa. Thus, only three out of the four candidates can be NE simultaneously. The exact number of NE can be determined following the method described in the following section, but it requires the complete knowledge of the channel gains. Proposition 1 aims at providing an estimation based only on the parameters $K$ and $S$.

\subsubsection{Determination of the NE}

In order to fully identify the action profiles corresponding to an NE, we use the graph $G$ described in the proof of Proposition 1 in Appendix. Basically, we convert the non-directed graph $G$ into an oriented graph $\hat{G}$ whose adjacency matrix is the non-symmetric square matrix $\hat{A}$ whose entries are $\forall(i, j) \in \mathcal{I}^{2}$ and $i \neq j$,

$$
\hat{a}_{i j}=\left\{\begin{array}{cc}
1 & \text { if } \quad i \in \mathcal{V}_{j} \text { and } \phi\left(p^{(j)}\right)>\phi\left(p^{(i)}\right) \\
0 & \text { otherwise, }
\end{array}\right.
$$

and $\hat{a}_{i, i}=0$ for all $i \in \mathcal{I}$. The set $\mathcal{V}_{j}$ contains the indices of all the vector that can be obtained by letting only one player to deviate from the action profile $\boldsymbol{p}^{(j)}$. This set is 
also called the neighborhood of action profile $\boldsymbol{p}^{(j)}$. More specifically, in the oriented graph $\hat{G}$, there exists an edge or arrow starting in vertex $v_{i}$ and ending in vertex $v_{j}$, i.e., $\hat{a}_{i j}=1$, if and only if $\phi\left(\boldsymbol{p}^{(j)}\right)>\phi\left(\boldsymbol{p}^{(i)}\right)$ and $\phi\left(\boldsymbol{p}^{(i)}\right)$ is in the neighborhood of $\phi\left(\boldsymbol{p}^{(j)}\right)$. Note that the first condition for adjacency in $\hat{G}$ represents the assumption of rational players. That is, a player changes its strategy if the new strategy brings a higher utility, i.e., it increases the potential function. The second condition enforces the fact that only one player can deviate at a time from the initial action profile $\boldsymbol{p}^{(i)}$. In Figure 1, we show an example of the non-directed $G$ graph and how it is transformed into an oriented graph $\hat{G}$ for the case where $K=3$ and $S=2$.

From the definition of the matrix $\hat{A}$, we have that a necessary and sufficient condition for a vertex $v_{i}$ to represent an NE action profile is to have a null out-degree in the oriented graph $\hat{G}$, i.e., there are no outgoing edges from the node $v_{i}$ (sink vertex). Finally, one can conclude that determining the set of NE in the game $\mathcal{G}_{(b)}$ boils down to identifying all the sink vertices in the oriented graph $\hat{G}$. Note that this method can be used only to determine the whole set of NE. It does not pretend to be an algorithm which can be directly implemented in decentralized wireless networks, since it requires complete information at each transmitter. Methods for achieving equilibria in wireless networks are described in $[7,8,13,14,30]$.

\section{Equilibrium performance analysis and special cases}

In this section, we study in detail two special cases of relevant interest to understand previous conclusions and provide more insights into decentralized PA problem in terms of design. First, the games $\mathcal{G}_{(a)}$ and $\mathcal{G}_{(b)}$ are studied assuming that there exist only $K=2$ transmitters and $S=2$ available channels. In particular, we analyze the set of NE action profiles of both games and compare the NSE,
$U^{(i)}: \mathcal{P}^{(i)} \rightarrow \mathbb{R}$, obtained by playing both games. Here, for all $i \in\{1,2\}$,

$$
U^{(i)}\left(\boldsymbol{p}_{1}, \ldots, \boldsymbol{p}_{K}\right)=\sum_{k=1}^{K} u_{k}\left(\boldsymbol{p}_{1}, \ldots, \boldsymbol{p}_{K}\right)[\mathrm{bps} / \mathrm{Hz}] .
$$

From this analysis, we conclude that limiting the transmitters to use a single channel brings a better result in terms of NSE (15). Second, we consider the case of a large number of transmitters. This study leads to two important conclusions: (i) the fraction of players using a given channel depends mainly on the bandwidth of each channel and not on the exact channel realization nor the number of players and channels; (ii) in the asymptotic regime $(K \rightarrow$ $\infty$ ) both games exhibit the same performance. Before we start, let us introduce the notion of best response correspondence, since it plays a central role in the following analysis.

Definition 3 (Best-response correspondence). In a non-cooperative game described by the 3-tuple $\left(\mathcal{K},\left(\mathcal{P}_{k}\right)_{\forall k \in \mathcal{K}},\left(u_{k}\right)_{\forall k \in \mathcal{K}}\right)$, the relation $B R_{k}: \mathcal{P}_{-k} \rightarrow \mathcal{P}_{k}$ such that

$$
B R_{k}\left(\boldsymbol{p}_{-k}\right)=\arg \max _{\boldsymbol{q}_{k} \in \mathcal{P}_{k}} u_{k}\left(\boldsymbol{q}_{k}, \boldsymbol{p}_{-k}\right)
$$

is defined as the best-response correspondence of player $k \in$ $\mathcal{K}$, given the actions $\boldsymbol{p}_{-k}$ adopted by all the other players.

\subsection{The 2-transmitter 2-channel case}

Consider the games $\mathcal{G}_{(a)}$ and $\mathcal{G}_{(b)}$ with $K=2$ and $S=2$. Assume also that $\forall k \in \mathcal{K}, p_{k, \max }=p_{\max }$ and $\forall s \in \mathcal{S}$, $\sigma_{s}^{2}=\sigma^{2}$ and $B_{s}=\frac{B}{S}$. Denote by SNR $=\frac{p_{\max }}{\sigma^{2}}$ the average signal-to-noise ratio (SNR) of each active communication.
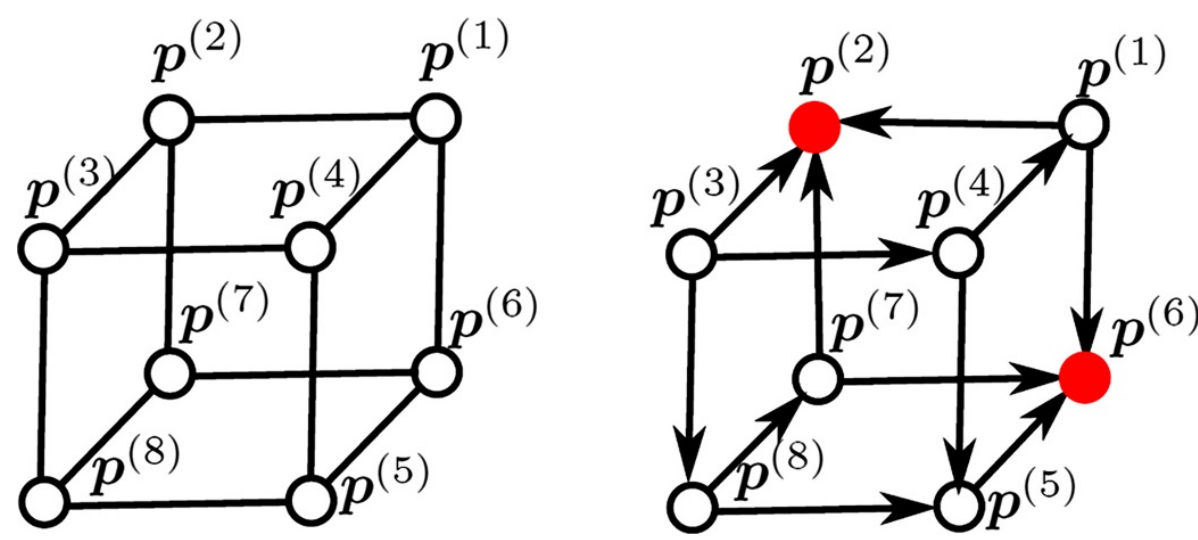

Figure 1 (a) Non-oriented graph and (b) oriented graph representing the game $\boldsymbol{G}_{(\boldsymbol{b})}$. The number of players is $K=3$ and the number of actions is $S=2$, under the condition $\phi\left(\boldsymbol{p}^{(\mathbf{2})}\right)>\phi\left(\boldsymbol{p}^{(\mathbf{6})}\right)>\phi\left(\boldsymbol{p}^{(\mathbf{1})}\right)>\phi\left(\boldsymbol{p}^{(\mathbf{5})}\right)>\phi\left(\boldsymbol{p}^{(\mathbf{4})}\right)>\phi\left(\boldsymbol{p}^{(7)}\right)>\phi\left(\boldsymbol{p}^{(\mathbf{8})}\right)>\phi\left(\boldsymbol{p}^{(\mathbf{3})}\right)$. Total number of vertices: $S^{K}=8$, number of neighbors per vertex: $K(S-1)=3$. Maximum Number of NE: 4. Number of NE: 2 (red vertices in (b)). 


\subsubsection{The PA game}

Let us denote by $\boldsymbol{p}^{\dagger}=\left(\boldsymbol{p}_{1}^{\dagger}, \boldsymbol{p}_{2}^{\dagger}\right)$ the NE of the game $\mathcal{G}_{(a)}$. Then, following Definition 1, one can write the following set of inclusions,

$$
\forall k \in \mathcal{K}, \quad \boldsymbol{p}_{k}^{\dagger} \in \mathrm{BR}_{k}\left(\boldsymbol{p}_{-k}^{\dagger}\right)
$$

Note that, for all $k \in \mathcal{K}$ and for all $\boldsymbol{p}_{-k} \in \mathcal{P}^{(a)}$, the set $\mathrm{BR}_{k}\left(\boldsymbol{p}_{-k}\right)$ is a singleton (Definition 3 ) and thus, (17) represents a system of equations. By solving the resulting system of equations (17) for a given realization of the channels $\left\{g_{i j}\right\}_{\forall(i, j) \in \mathcal{K} \times \mathcal{S}}$, one can determine the NE of the game $\mathcal{G}_{(a)}$. We present such a solution in the following proposition.

Proposition $2\left(\mathrm{NE}\right.$ in $\left.\boldsymbol{G}_{(\boldsymbol{a})}\right)$. Let the action profile $\boldsymbol{p}^{\dagger}=$ $\left(\boldsymbol{p}_{1}^{\dagger}, \boldsymbol{p}_{2}^{\dagger}\right) \in \mathcal{P}^{(a)}$, with $\boldsymbol{p}_{1}^{\dagger}=\left(p_{11}^{\dagger}, p_{\max }-p_{11}^{\dagger}\right)$ and $\boldsymbol{p}_{2}^{\dagger}=$ $\left(p_{\max }-p_{22}^{\dagger}, p_{22}^{\dagger}\right)$ be an NE action profile of the game $\mathcal{G}_{(a)}$ with channel realizations $\boldsymbol{g}=\left(g_{11}, g_{12}, g_{21}, g_{22}\right)$. Then, with probability one, $\boldsymbol{p}^{\dagger}$ is the unique NE and it can be written as follows:

- Equilibrium 1: if $\boldsymbol{g} \in \mathcal{A}_{1}=\left\{\boldsymbol{g} \in \mathbb{R}_{+}^{4}: \frac{g_{11}}{g_{12}} \geqslant \frac{1+S N R g_{11}}{1+S N R g_{22}}\right.$, $\left.\frac{g_{21}}{g_{22}} \leqslant \frac{1+S N R g_{11}}{1+S N R g_{22}}\right\}$, then, $p_{11}^{\dagger}=p_{\max }$ and $p_{22}^{\dagger}=p_{\max }$.

- Equilibrium 2: if $\boldsymbol{g} \in \mathcal{A}_{2}=\left\{\boldsymbol{g} \in \mathbb{R}_{+}^{4}: \frac{g_{11}}{g_{12}} \geqslant 1+\right.$ $\left.\operatorname{SNR}\left(g_{11}+g_{21}\right), \frac{g_{21}}{g_{22}} \geqslant 1+\operatorname{SNR}\left(g_{11}+g_{21}\right)\right\}$, then, $p_{11}^{\dagger}=p_{\max }$ and $p_{22}^{\dagger}=0$.

- Equilibrium 3: if $\boldsymbol{g} \in \mathcal{A}_{3}=\left\{\boldsymbol{g} \in \mathbb{R}_{+}^{4}: \frac{g_{11}}{g_{12}} \leqslant\right.$ $\left.\frac{1}{1+S N R\left(g_{12}+g_{22}\right)}, \frac{g_{21}}{g_{22}} \leqslant \frac{1}{1+S N R\left(g_{12}+g_{22}\right)}\right\}$ then, $p_{11}^{\dagger}=0$ and $p_{22}^{\dagger}=p_{\max }$.

- Equilibrium 4: if $\boldsymbol{g} \in \mathcal{A}_{4}=\left\{\boldsymbol{g} \in \mathbb{R}_{+}^{4}: \frac{g_{11}}{g_{12}} \leqslant \frac{1+S N R g_{21}}{1+S N R g_{12}}\right.$, $\left.\frac{g_{21}}{g_{22}} \geqslant \frac{1+\operatorname{SNRg}_{21}}{1+S N R g_{12}}\right\}$, then, $p_{11}^{\dagger}=0$ and $p_{22}^{\dagger}=0$.

- Equilibrium 5: if $\boldsymbol{g} \in \mathcal{A}_{5}=\left\{\boldsymbol{g} \in \mathbb{R}_{+}^{4}: \frac{g_{11}}{g_{12}} \geqslant \frac{g_{21}}{g_{22}}\right.$, $\left.\frac{1+S N R g_{11}}{1+S N R g_{22}}<\frac{g_{21}}{g_{22}}<1+\operatorname{SNR}\left(g_{11}+g_{21}\right)\right\}$, then, $p_{11}^{\dagger}=p_{\max }$ and $p_{22}^{\dagger}=\frac{1}{2}\left(p_{\max }-\frac{\sigma^{2}}{g_{22}}+\frac{\sigma^{2}+g_{11} p_{\max }}{g_{21}}\right)$.

- Equilibrium 6: if $\boldsymbol{g} \in \mathcal{A}_{6}=\left\{\boldsymbol{g} \in \mathbb{R}_{+}^{4}: \frac{g_{11}}{g_{12}} \geqslant \frac{g_{21}}{g_{22}}\right.$, $\left.\frac{1}{1+\operatorname{SNR}\left(g_{12}+g_{22}\right)}<\frac{g_{11}}{g_{12}}<\frac{1+\operatorname{SNRg}_{11}}{1+S N R g_{22}}\right\}$, then, $p_{11}^{\dagger}=\frac{1}{2}\left(p_{\max }-\frac{\sigma^{2}}{g_{11}}+\frac{\sigma^{2}+p_{\max } g_{22}}{g_{12}}\right)$ and $p_{22}^{\dagger}=p_{\max }$.

- Equilibrium 7: if $\boldsymbol{g} \in \mathcal{A}_{7}=\left\{\boldsymbol{g} \in \mathbb{R}_{+}^{4}: \frac{g_{11}}{g_{12}} \leqslant \frac{g_{21}}{g_{22}}\right.$, $\left.\frac{1+S N R g_{21}}{1+S N R g_{12}}<\frac{g_{11}}{g_{12}}<1+\operatorname{SNR}\left(g_{11}+g_{21}\right)\right\}$, then, $p_{11}^{\dagger}=\frac{1}{2}\left(p_{\max }-\frac{\sigma^{2}+p_{\max } g_{21}}{g_{11}}+\frac{\sigma^{2}}{g_{12}}\right)$ and $p_{22}^{\dagger}=0$.

- Equilibrium 8: if $\boldsymbol{g} \in \mathcal{A}_{8}=\left\{\boldsymbol{g} \in \mathbb{R}_{+}^{4}: \frac{g_{11}}{g_{12}} \leqslant \frac{g_{21}}{g_{22}}\right.$, $\left.\frac{1}{1+S N R\left(g_{12}+g_{22}\right)}<\frac{g_{21}}{g_{22}}<\frac{1+S N R g_{21}}{1+S N R g_{12}}\right\}$, then, $p_{11}^{\dagger}=0$ and $p_{22}^{\dagger}=\frac{1}{2}\left(p_{\max }-\frac{\sigma^{2}+g_{12} p_{\max }}{g_{22}}+\frac{\sigma^{2}}{g_{21}}\right)$.
The proof of Proposition 2 is given in Appendix.

In Figure 2, we plot the different types of NE of the game $\mathcal{G}_{(a)}$ as a function of the channel ratios $\frac{g_{11}}{g_{12}}$ and $\frac{g_{21}}{g_{22}}$. Note that under the knowledge of all channels, the set of all possible pure NE can be obtained by simply placing the point $\left(\frac{g_{11}}{g_{12}}, \frac{g_{21}}{g_{22}}\right)$ in Figure 2. The uniqueness of the NE is not ensured under certain conditions. In fact, infinitely many NE can be observed, however, the conditions for this to happen are zero probability events, as we shall see in the following lemma.

Lemma 4. Let $\alpha \triangleq \frac{g_{11}}{g_{21}}=\frac{g_{12}}{g_{22}}$ and assume that the set of channels $\left\{g_{i j}\right\}_{\forall(i, j) \in \mathcal{K} \times \mathcal{S}}$ satisfies the following conditions

$$
\frac{1}{1+\frac{p_{\max }}{\sigma^{2}}\left(g_{12}+g_{22}\right)}<\alpha<1+\frac{p_{\max }}{\sigma^{2}}\left(g_{11}+g_{21}\right)
$$

Then, any $P A$ vector $\boldsymbol{p}=$ $\left(p_{11}, p_{\max }-p_{11}, p_{\max }-p_{22}, p_{22}\right) \in \mathcal{P}^{(a)}$, such that

$$
p_{11}=\frac{1}{2}\left(p_{\max }(1-\alpha)+\sigma^{2}\left(\frac{1}{g_{12}}-\frac{1}{g_{11}}\right)\right)+\alpha p_{22}
$$

is an NE action profile of the game $\mathcal{G}_{(a)}$.

The proof of Lemma 4 is the first part of the proof of Proposition 2. In the following section, we perform the same analysis presented above for the game $\mathcal{G}_{(b)}$.

\subsubsection{The CS game}

When $K=2$ and $S=2$, the game $\mathcal{G}_{(b)}$ has four possible outcomes, i.e., $\left|\mathcal{P}^{(b)}\right|=4$. We detail such outcomes and its respective potential in Table 1. Following Definition 1, each of those outcomes can be an NE depending on the channel realizations $\left\{g_{i j}\right\}_{\forall(i, j) \in \mathcal{K} \times \mathcal{S}}$, as shown in the following proposition.

Proposition $3\left(\mathrm{NE}\right.$ in $\left.\boldsymbol{G}_{(b)}\right)$. Let the PA vector $\boldsymbol{p}^{*}=$ $\left(\boldsymbol{p}_{1}^{*}, \boldsymbol{p}_{2}^{*}\right) \in \mathcal{P}^{(b)}$ be one NE in the game $\mathcal{G}_{(b)}$. Then, depending on the channel gains $\boldsymbol{g}=\left(g_{11}, g_{12}, g_{21}, g_{22}\right)$, the $N E \boldsymbol{p}^{*}$ can be written as follows:

- Equilibrium 1: when $\boldsymbol{g} \in \mathcal{B}_{1}=\left\{\boldsymbol{g} \in \mathbb{R}_{+}^{4}: \frac{g_{11}}{g_{12}} \geqslant\right.$ $\frac{1}{1+S N R g_{22}}$ and $\left.\frac{g_{21}}{g_{22}} \leqslant 1+S N R g_{11}\right\}$, then, $\boldsymbol{p}_{1}^{*}=\left(p_{\max }, 0\right)$ and $\boldsymbol{p}_{2}^{*}=\left(0, p_{\max }\right)$.

- Equilibrium 2: When $\boldsymbol{g} \in \mathcal{B}_{2}=\left\{\boldsymbol{g} \in \mathbb{R}_{+}^{4}: \frac{g_{11}}{g_{12}} \geqslant 1+\right.$ $S N R g_{21}$ and $\left.\frac{g_{21}}{g_{22}} \geqslant 1+S N R g_{11}\right\}$, then, $\boldsymbol{p}_{1}^{*}=\left(p_{\max }, 0\right)$ and $\boldsymbol{p}_{2}^{*}=\left(p_{\max }, 0\right)$.

- Equilibrium 3: when $\boldsymbol{g} \in \mathcal{B}_{3}=\left\{\boldsymbol{g} \in \mathbb{R}_{+}^{4}: \frac{g_{11}}{g_{12}} \leqslant\right.$ $\frac{1}{1+S N R g_{22}}$ and $\left.\frac{g_{21}}{g_{22}} \leqslant \frac{1}{1+S N R g_{12}}\right\}$, then, $\boldsymbol{p}_{1}^{*}=\left(0, p_{\max }\right)$ and $\boldsymbol{p}_{2}^{*}=\left(0, p_{\max }\right)$. 


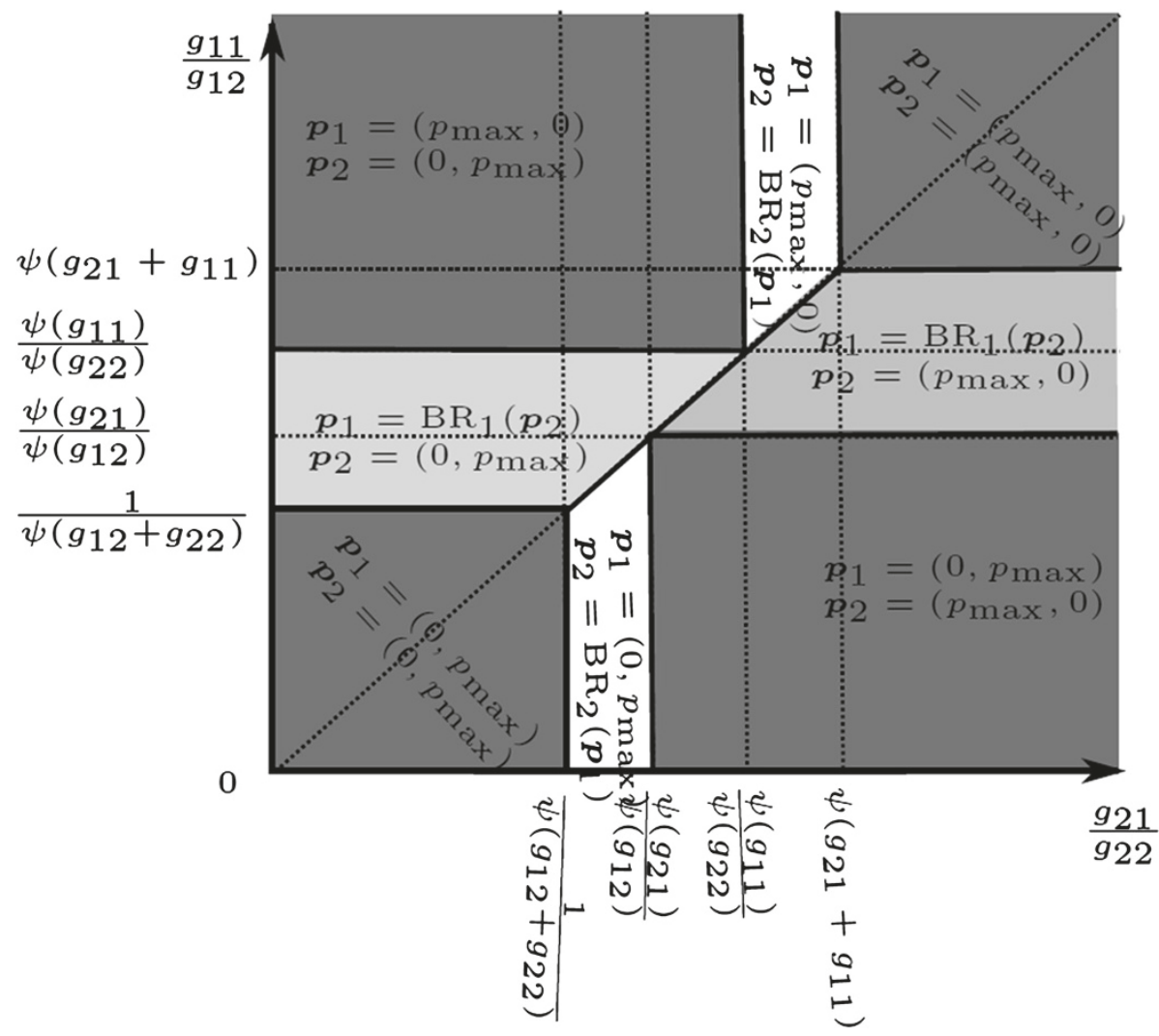

Figure 2 Nash equilibrium action profiles as a function of the channel ratios $\frac{g_{11}}{g_{12}}$ and $\frac{g_{21}}{g_{22}}$ for the two-player-two-channel game $G_{(a)}$. The function $\psi: \mathbb{R}_{+} \rightarrow \mathbb{R}_{+}$is defined as follows: $\psi(x)=1+\operatorname{SNR} x$. The best response function $\operatorname{BR}_{k}\left(\boldsymbol{p}_{-k}\right)$, for all $k \in \mathcal{K}$, is defined by (12). Here, it has been arbitrarly assumed that $\frac{\psi\left(g_{21}\right)}{\psi\left(g_{12}\right)}<\frac{\psi\left(g_{11}\right)}{\psi\left(g_{22}\right)}$.

- Equilibrium 4: when $\boldsymbol{g} \in \mathcal{B}_{4}=\left\{\boldsymbol{g} \in \mathbb{R}_{+}^{4}: \frac{g_{11}}{g_{12}} \leqslant 1+\right.$ $S N R g_{12}$ and $\left._{g_{22}} g_{21} \geqslant \frac{1}{1+S N R g_{12}}\right\}$, then, $\boldsymbol{p}_{1}^{*}=\left(0, p_{\max }\right)$ and $\boldsymbol{p}_{2}^{*}=\left(p_{\max }, 0\right)$.

The proof follows immediately from Definition 1 and Table 1. In Figure 3, we plot the different types of NE action profiles as a function of the channel ratios $\frac{g_{11}}{g_{12}}$

Table 1 Potential function $\phi$ of the game $G_{(a)}$, with $K=2$ and $S=2$

\begin{tabular}{lcc}
\hline $\boldsymbol{T}_{\boldsymbol{x}_{\mathbf{1}} \mid \boldsymbol{T}_{\boldsymbol{x}_{\mathbf{2}}}}$ & $\boldsymbol{p}_{\mathbf{2}}=\left(\boldsymbol{p}_{\text {max }}, \mathbf{0}\right)$ & $\boldsymbol{p}_{\mathbf{2}}=\left(\mathbf{0}, \boldsymbol{p}_{\text {max }}\right)$ \\
\hline $\boldsymbol{p}_{1}=\left(p_{\max }, 0\right)$ & $\frac{1}{2} \log _{2}\left(\sigma^{2}+p_{\max }\left(g_{11}+g_{21}\right)\right)$ & $\frac{1}{2} \log _{2}\left(\sigma^{2}+p_{\max } g_{11}\right)$ \\
& $+\frac{1}{2} \log _{2}\left(\sigma^{2}\right)$ & $+\frac{1}{2} \log _{2}\left(\sigma^{2}+p_{\max } g_{22}\right)$ \\
\hline $\boldsymbol{p}_{1}=\left(0, p_{\max }\right)$ & $\frac{1}{2} \log _{2}\left(\sigma^{2}+p_{\max } g_{12}\right)$ & $\frac{1}{2} \log _{2}\left(\sigma^{2}+p_{\max }\left(g_{12}+g_{22}\right)\right)$ \\
& $+\frac{1}{2} \log _{2}\left(\sigma^{2}+p_{\max } g_{21}\right)$ & $+\frac{1}{2} \log _{2}\left(\sigma^{2}\right)$ \\
\hline
\end{tabular}

Player 1 chooses rows and player 2 chooses columns. and $\frac{g_{21}}{g_{22}}$. Note that under the knowledge of all channels, the set of all possible pure NE can be obtained by simply placing the point $\left(\frac{g_{11}}{g_{12}}, \frac{g_{21}}{g_{22}}\right)$ in Figure 3 . Note how the action profiles $\boldsymbol{p}^{*}=\left(p_{\max }, 0,0, p_{\max }\right)$ and $\boldsymbol{p}^{+}=\left(0, p_{\max }, p_{\max }, 0\right)$ are both NE, when the channel realizations satisfy that $g \in \mathcal{B}_{5}=\mathcal{B}_{1} \cap \mathcal{B}_{4}$, i.e.,

$$
\begin{gathered}
\mathcal{B}_{5}=\left\{g \in \mathbb{R}_{+}^{4}: \frac{1}{1+\mathrm{SNR}_{22}} \leqslant \frac{g_{11}}{g_{12}} \leqslant 1+\mathrm{SNR}_{21}\right. \text { and } \\
\left.\frac{1}{1+\mathrm{SNR} g_{12}} \leqslant \frac{g_{21}}{g_{22}} \leqslant 1+\mathrm{SNR} g_{11}\right\} .
\end{gathered}
$$

This confirms the fact that several NE might exist in the game $\mathcal{G}_{(b)}$ depending on the exact channel realization, as stated in Proposition 1. Moreover, one can also observe that there might exist an NE action profile which is not a global maximizer of the potential function (9) [33] (e.g., $\left.\phi\left(\boldsymbol{p}^{*}\right)<\phi\left(\boldsymbol{p}_{2}^{+}\right)\right)$. 


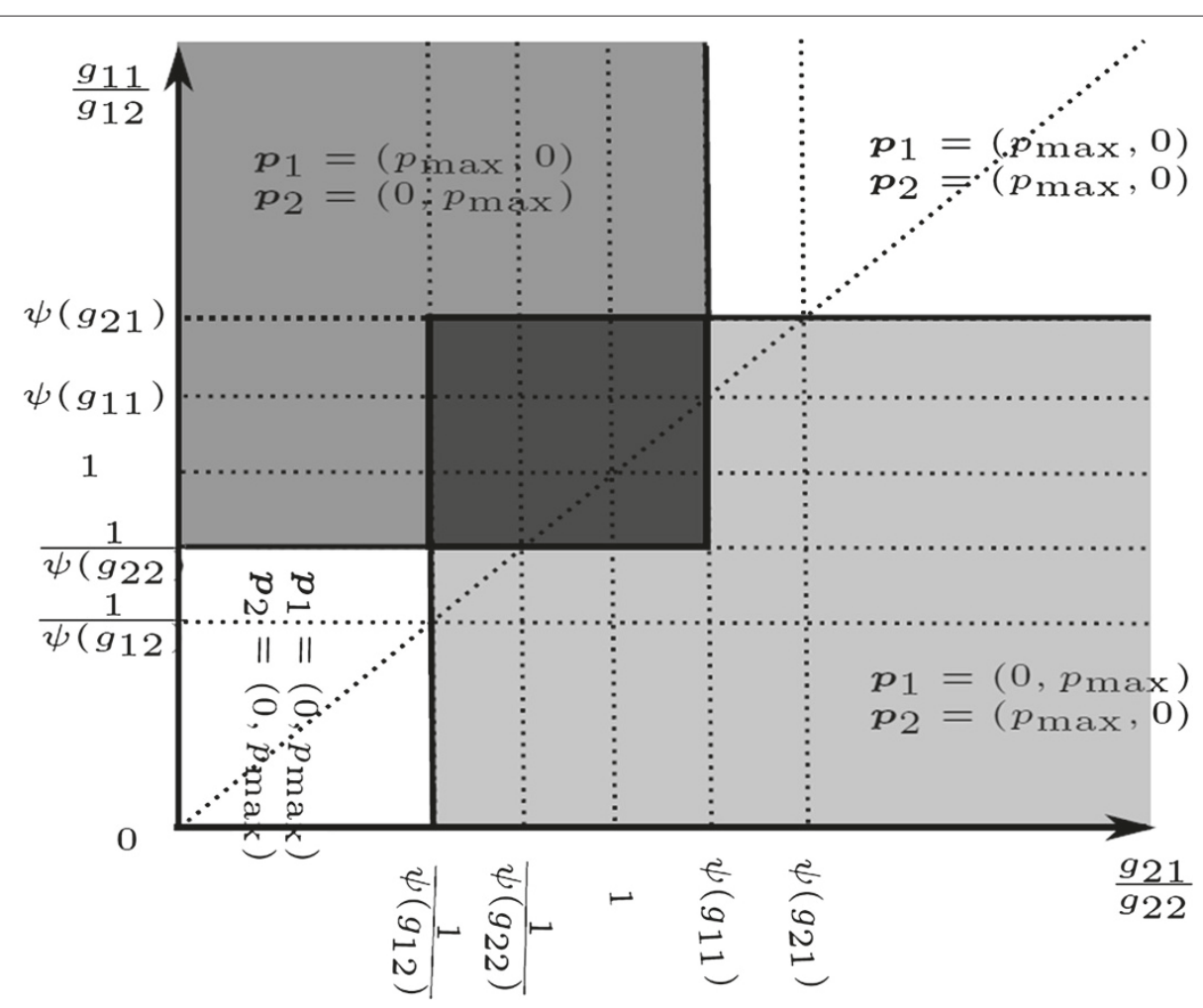

Figure 3 Nash equilibrium action profiles as a function of the channel ratios $\frac{g_{11}}{g_{12}}$ and $\frac{g_{21}}{g_{22}}$ for the two-player-two-channel game $G_{(b)}$, respectively. The function $\psi: \mathbb{R}_{+} \rightarrow \mathbb{R}_{+}$is defined as follows: $\psi(x)=1+\operatorname{SNR} x$. The best response function $B R_{k}\left(\boldsymbol{p}_{-k}\right)$, for all $k \in \mathcal{K}$, is defined by (12). Here, it has been arbitrarly assumed that $\psi\left(g_{11}\right)<\psi\left(g_{21}\right)$.

In the sequel, the performance achieved by the transmitters at the equilibrium in both games are compared.

\subsection{A Braess-type paradox}

As suggested in [23], a Braess-type paradox refers to a counter-intuitive observation consisting in a reduction of the individual utility at the equilibria, when the players are granted with a larger set of actions. That is, by letting the players to choose from a larger set of options, their individual benefit reduces. Recently, the Braess-type paradox has been also associated with the reduction of the sumutility instead of the individual utilities, as in $[20,25,34]$ in the wireless communications arena.

In our particular case, the set of actions for player $k$ in the game $\mathcal{G}_{(b)}$ is a subset of its set of actions in the game $\mathcal{G}_{(a)}$, i.e., $\forall k \in \mathcal{K}, \mathcal{P}_{k}^{(b)} \subseteq \mathcal{P}_{k}^{(a)}$. Interestingly, as observed in [23], reducing the set of actions of each player leads, in this particular game, to a better global performance. This effect has been reported in the parallel interference channel in $[24,25]$ under the consideration of particular channel conditions and later, more generally in [20]. However, a formal proof of the existence of this paradox is not provided in the aforementioned references. This observation has also been reported in the parallel MAC for the case of successive interference cancellation (SIC) in [35]. Nonetheless, the channel in [35] was not fully decentralized, as it required a central controller to dictate the channel policy to all the transmitters. In the following, we study this observation in more detail.

Let us denote by $\boldsymbol{p}_{k}^{(\dagger, n)}$ the unique NE action profile of game $\mathcal{G}_{(a)}$, when the vector $\boldsymbol{g}=\left(g_{11}, g_{12}, g_{21}, g_{22}\right) \in \mathcal{A}_{n}$, for all $n \in\{1 \ldots, 8\}$. Let us also denote by $\boldsymbol{p}^{(*, n)}$ one of the $\mathrm{NE}$ action profiles of game $\mathcal{G}_{(b)}$ when $\left(g_{11}, g_{12}, g_{21}, g_{22}\right) \in$ $\mathcal{B}_{n}$, for all $n \in\{1, \ldots 4\}$. The sets $\mathcal{A}_{n}$ and $\mathcal{B}_{n}$ are defined in Propositions 2 and 3. Then, for a finite SNR level, one can observe that $\forall n \in\{1, \ldots, 4\}, \mathcal{A}_{n} \cap \mathcal{B}_{n}=\mathcal{A}_{n}$ and $\forall \boldsymbol{g}=\left(g_{11}, g_{12}, g_{21}, g_{22}\right) \in \mathcal{A}_{n}$, the following equality always holds $\boldsymbol{p}_{k}^{(\dagger, n)}=\boldsymbol{p}_{k}^{(*, n)}$, which implies the same network performance. However, when the NE of both games are different, one cannot easily compare the utilities achieved by each player since they depend on the exact channel realizations. Fortunately, the analysis largely simplifies by considering either a low SNR regime or a high SNR regime and more general conclusions can be stated. The performance comparison between games $\mathcal{G}_{(a)}$ and $\mathcal{G}_{(b)}$ for the low SNR regime is presented in the following proposition. 
Proposition 4. In the low SNR regime, both games $\mathcal{G}_{(a)}$ and $\mathcal{G}_{(b)}$, with $K=2$ and $S=2$, possess a unique $N E$, denoted by $\boldsymbol{p}^{*}$. Here, for all $k \in \mathcal{K}$ and $s \in \mathcal{S}$,

$$
\begin{aligned}
p_{k, s}^{*} & =p_{\max } \mathbb{1}_{\left\{s=\arg \max _{\ell \in \mathcal{S}} g_{k, \ell}\right\}} \\
p_{k,-s}^{*} & =p_{\max }-p_{k, s} .
\end{aligned}
$$

The proof of Proposition 4 is given in Appendix. From, Proposition 4, it can be stated that at the low SINR regime, players achieve the NE by simply choosing the channel with the highest channel gain independently of the other player's action. The performance comparison between games $\mathcal{G}_{(a)}$ and $\mathcal{G}_{(b)}$ for the high SNR regime is presented in the following proposition.

Proposition 5. In the high SNR regime, with $K=2$ and $S=2$, the game $\mathcal{G}_{(\text {a) }}$ has a unique pure NE denoted by $\boldsymbol{p}^{\dagger}$ and the game $\mathcal{G}_{(b)}$ has two pure NE denoted by $\boldsymbol{p}^{(*, 1)}$ and $\boldsymbol{p}^{(*, 4)}$, respectively. Then, at least for one $n \in\{1,4\}$, there exists an $S N R$ value $0<S N R_{0}<\infty$, such that $\forall S N R \geqslant$ $S N R_{0}$,

$$
\sum_{k=1}^{2} u_{k}\left(\boldsymbol{p}^{(*, n)}\right)-\sum_{k=1}^{2} u_{k}\left(\boldsymbol{p}^{\dagger}\right) \geqslant \delta
$$

and $\delta \geqslant 0$.

The proof of Proposition 5 is given in Appendix. From the proof of Proposition 5, it can be stated that in none of the games, players transmit simultaneously on the same channels. Now, from Propositions 4 and 5, it can be concluded that at low SNR both games $\mathcal{G}_{(a)}$ and $\mathcal{G}_{(b)}$ induce the same NSE. On the contrary, the game $\mathcal{G}_{(b)}$ always induces a higher or equal NSE than the game $\mathcal{G}_{(a)}$ in the high SNR regime. This counter-intuitive result implies a Braess-type paradox, as suggested in the beginning of this section.

\subsection{The case of large systems}

In this section, we deal with the games $\mathcal{G}_{(b)}$ for the case of large networks, i.e., networks with a large number of transmitters. Within this scenario, the dominant parameter to analyze these games is the fraction of transmitters using a particular channel. As we shall see, contrary to the case of small number of transmitters and channels analyzed in the previous section, in the case of large networks, each player becomes indifferent to the action adopted by each of the other players. Here, each player is rather concerned with the fractions of players simultaneously playing the same action, i.e., using the same channel. Hence, one of the interesting issues to be solved is the determination of the repartition of the users between the different channels at the NE.

As a first step towards identifying the fractions of transmitters per channel at the NE, we first re-write the potential (9) as a function of the vector $\boldsymbol{x}(\boldsymbol{p})=$ $\left(x_{1}(\boldsymbol{p}), \ldots, x_{S}(\boldsymbol{p})\right)$, where $x_{s}(\boldsymbol{p})$, with $s \in \mathcal{S}$, denotes the fraction of players transmitting over channel $s$ given the action profile $\boldsymbol{p} \in \mathcal{P}^{(b)}$. Hence,

$$
\forall s \in \mathcal{S}, \begin{aligned}
x_{s}(\boldsymbol{p}) & =\frac{\left|\mathcal{K}_{s}(\boldsymbol{p})\right|}{K} \\
\sum_{i=1}^{S} x_{i}(\boldsymbol{p}) & =1,
\end{aligned}
$$

where $\mathcal{K}_{s}(\boldsymbol{p}) \subseteq \mathcal{K}$ is the set of players using channel $s$ given the action profile $\boldsymbol{p} \in \mathcal{P}^{(b)}$, i.e., $\mathcal{K}_{s}(\boldsymbol{p})=\left\{k \in \mathcal{K}: p_{k, s} \neq\right.$ $0\}$. Let $b_{s}=\frac{B_{s}}{B}$ denote the fraction of bandwidth associated with channel $s$, such that $\sum_{s=1}^{S} b_{s}=1$. Then, one can write the potential as follows

$$
\begin{aligned}
\phi(\boldsymbol{p})= & \sum_{s=1}^{S} b_{s} \log _{2}\left(N_{0} B_{s}+p_{\max } \sum_{k \in \mathcal{K}_{s}(\boldsymbol{p})} g_{k, s}\right) \\
= & S \log _{2}(K)+\sum_{s=1}^{S} b_{s} \log _{2}\left(\frac{N o B_{s}}{K}+x_{s}(\boldsymbol{p}) p_{\max }\right. \\
& \left.\times\left(\frac{1}{\left|\mathcal{K}_{s}(\boldsymbol{p})\right|} \sum_{k \in \mathcal{K}_{s}(\boldsymbol{p})} g_{k, s}\right)\right) .
\end{aligned}
$$

Note that the term $S \log _{2}(K)$ in (23) does not depend on the actions of the players. Thus, in the following, we drop it for the sake of simplicity. We assume that the number of players $K$ and the available bandwidth $B$ grows to infinite at a constant rate $\mu>0$, while the fractions $b_{s}$, for all $s \in \mathcal{S}$ are kept invariant. That is, the average bandwidth per transmitters is asymptotically constant,

$$
\lim _{K, B \rightarrow \infty} \frac{B}{K}=\mu \text {. }
$$

Thus, under the assumption of large number of transmitters and for any action profile $\boldsymbol{p} \in \mathcal{P}^{(b)}$, it follows that,

$$
\forall s \in \mathcal{S}, \frac{1}{\left|\mathcal{K}_{s}(\boldsymbol{p})\right|} \sum_{k \in \mathcal{K}_{s}(\boldsymbol{p})} g_{k, s} \stackrel{K \rightarrow \infty}{\longrightarrow} \int_{0}^{\infty} \lambda \mathrm{F}_{g_{s}}(\lambda)=\Omega_{s},
$$

where $F_{g_{s}}$ is the cumulative probability function associated with the channel gains over dimension $s$. Hence, for all action profile $\boldsymbol{p} \in \mathcal{P}^{(b)}$ adopted by the players, maximizing the function $\phi(\boldsymbol{p})$ in the asymptotic regime reduces to maximize the function $\tilde{\phi}(\boldsymbol{x}(\boldsymbol{p}))$,

$$
\tilde{\phi}(\boldsymbol{x}(\boldsymbol{p}))=\sum_{s=1}^{S} b_{s} \log _{2}\left(\mu N o b_{s}+x_{s}(\boldsymbol{p}) p_{\max } \Omega_{s}\right) .
$$

That is, solving the OP,

$$
\left\{\begin{array}{cc}
\max _{x=\left(x_{1}, \ldots, x_{S}\right) \in \mathbb{R}_{+}^{S}} & \sum_{s=1}^{S} b_{s} \log _{2}\left(\mu N_{0} b_{s}+x_{s} p_{\max } \Omega_{s}\right), \\
\text { s.t. } & \sum_{i=1}^{S} x_{i}=1 \text { and } \forall i \in \mathcal{S}, x_{i} \geqslant 0,
\end{array} .\right.
$$


The optimization problem above has a unique solution of the form,

$$
\forall s \in \mathcal{S}, \quad x_{s}=b_{s}\left[\frac{1}{\beta_{k}}-\frac{\mu N_{0}}{p_{\max } \Omega_{s}}\right]^{+},
$$

where $\beta_{k}$ is Lagrangian multiplier to satisfy the optimization constraints. Interestingly, in the case when all the channels are described with the same statistics, that is, $\forall s \in \mathcal{S}, F_{g_{s}}(\lambda)=F_{g}(\lambda),\left(\forall s \in \mathcal{S}, \Omega_{s}=\Omega\right)$ it holds that,

$$
\forall s \in \mathcal{S}, \quad x_{s}=\frac{B_{s}}{B} .
$$

The above provides a very simple relation between the repartition of the users among the available channels in the asymptotic regime. Indeed, it can be implied that the number of transmitters using a given channel $s$ is proportional to the bandwidth allocated to the corresponding channel. In particular, note that this result generalizes the work in [36].

To conclude on the usefulness of the large system analysis, let us consider the upper bound on the number of $\mathrm{NE}$ which is given by Proposition 1 . Let us normalize the upper bound on the number of pure NE $L$ in (13) by the total number of pure (CS) strategy profiles, and let us write,

$$
\frac{L}{S^{K}}<\frac{1}{S^{K}}\left(1+(S-1) 2^{K}\right) .
$$

Now, for a sufficiently large number $K$, the following approximation holds,

$$
\frac{1}{S^{K}}\left(1+(S-1) 2^{K}\right) \approx(S-1)\left(\frac{2}{S}\right)^{K} .
$$

Although the number of pure NE in the CS game might be conjectured to be large, it is in fact relatively small in the asymptotic regime. Indeed, (28) indicates that when the number of users is large, the fraction of pure NE goes to zero whenever the number of channels is greater or equal to 3 . This result shows the difficulty of using methodologies such as the one proposed in Section 3.3.3 to study the set of NE or the difficulty of achieving equilibria using decentralized learning algorithms as proposed in $[7,8]$.

\subsection{PoA and PoS}

The PoA [37] and the $\operatorname{PoS}^{\mathrm{a}}$ [38] are both measures of the efficiency of the set of equilibria of a game. Basically, the PoA measures the loss of global performance due to decentralization by comparing the maximum sum utility achieved under the global control of a central authority with the minimum sum utility achieved at the Nash equilibrium. The PoS measures also the loss of global performance due to decentralization by comparing the maximum sum utility achieved under the global control of an authority with the maximum sum utility achieved at the Nash equilibrium. For the PA game $\mathcal{G}_{(a)}$ and the CS game $\mathcal{G}_{(b)}$, the PoA and the PoS can be written as follows,

$$
\operatorname{PoA}\left(\mathcal{G}_{(i)}\right)=\frac{\max _{\boldsymbol{p} \in \mathcal{P}^{(i)}} U^{(i)}(\boldsymbol{p})}{\min _{\boldsymbol{p} \in \mathcal{P}_{\mathrm{NE}}^{(i)}} U^{(i)}(\boldsymbol{p})},
$$

and

$$
\operatorname{PoS}\left(\mathcal{G}_{(i)}\right)=\frac{\max _{\boldsymbol{p} \in \mathcal{P}^{(i)}} U^{(i)}(\boldsymbol{p})}{\max _{\boldsymbol{p} \in \mathcal{P}_{\mathrm{NE}}^{(i)}} U^{(i)}(\boldsymbol{p})},
$$

respectively. Here, $i \in\{a, b\}, U^{(i)}$ is defined in (15) and $\mathcal{P}_{\mathrm{NE}}^{(i)}$ is the set of NE of the game $\mathcal{G}_{(i)}$. Regarding the optimization problem in the numerator of (29) and (30), we have that the following holds from the information theoretical upper bound of the sum of individual rates of the MAC [1]:

$$
\begin{aligned}
\sum_{k=1}^{K} u_{k}(\boldsymbol{p}) \stackrel{(a)}{\leqslant} & \sum_{s \in \mathcal{S}} \frac{B_{s}}{B} \log _{2}\left(1+\frac{\sum_{k=1}^{K} p_{k, s} g_{k, s}}{\sigma_{s}^{2}}\right) \\
= & \sum_{s \in \mathcal{S}} \frac{B_{s}}{B} \log _{2}\left(\sigma_{s}^{2}+\sum_{k \in \mathcal{K}} p_{k, s} g_{k, s}\right) \\
& -\sum_{s \in \mathcal{S}} \frac{B_{s}}{B} \log _{2}\left(\sigma_{s}^{2}\right), \\
= & \phi(\boldsymbol{p})-\sum_{s \in \mathcal{S}} \frac{B_{s}}{B} \log _{2}\left(\sigma_{s}^{2}\right), \\
& \stackrel{(b)}{\leqslant} \phi\left(\boldsymbol{p}^{\ddagger}\right)-\sum_{s \in \mathcal{S}} \frac{B_{s}}{B} \log _{2}\left(\sigma_{s}^{2}\right) .
\end{aligned}
$$

Here, (a) holds with strict equality, for any given PA vector $\boldsymbol{p}$, when the receiver implements (perfect) SIC. Moreover, $(b)$ holds with strict equality when (perfect) SIC is used at the receiver and $\boldsymbol{p}^{\ddagger}$ is the PA vector at the NE with the highest NSE. That is,

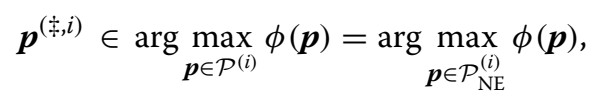

where the equality in (35) holds since the maximizers of the function $\phi$ are the set of NE as shown in Section 3.

Regarding the optimization problem in the denominator of (29) and (30), we use the following notation

$$
\begin{aligned}
& \boldsymbol{p}^{(\top, i)} \in \arg \max _{\boldsymbol{p} \in \mathcal{P}_{\mathrm{NE}}^{(i)}} U^{(i)}(\boldsymbol{p}) \text { and } \\
& \boldsymbol{p}^{(\perp, i)} \in \arg \min _{\boldsymbol{p} \in \mathcal{P}_{\mathrm{NE}}^{(i)}} U^{(i)}(\boldsymbol{p}) .
\end{aligned}
$$


and we write (29) and (30) as follows,

$$
\begin{aligned}
& \operatorname{PoA}\left(\mathcal{G}_{(i)}\right) \\
& =\frac{\sum_{s \in \mathcal{S}} \frac{B_{s}}{B} \log _{2}\left(\sigma_{s}^{2}+\sum_{k=1}^{K} p_{k, s}^{(\ddagger, i)} g_{k, s}\right)-\sum_{s \in \mathcal{S}} \frac{B_{s}}{B} \log _{2}\left(\sigma_{s}^{2}\right)}{\sum_{k=1}^{K} u_{k}\left(\boldsymbol{p}^{(\perp, i)}\right)},
\end{aligned}
$$

and

$$
\begin{aligned}
& \operatorname{PoS}\left(\mathcal{G}_{(i)}\right) \\
& =\frac{\sum_{s \in \mathcal{S}} \frac{B_{s}}{B} \log _{2}\left(\sigma_{s}^{2}+\sum_{k=1}^{K} p_{k, s}^{(\dagger, i)} g_{k, s}\right)-\sum_{s \in \mathcal{S}} \frac{B_{s}}{B} \log _{2}\left(\sigma_{s}^{2}\right)}{\sum_{k=1}^{K} u_{k}\left(\boldsymbol{p}^{(\top, i)}\right)},
\end{aligned}
$$

respectively.

Now, using the fact that, for all $\boldsymbol{p} \in \mathcal{P}^{(a)}$,

$$
\begin{aligned}
& \sum_{s \in \mathcal{S}} \frac{B_{s}}{B} \log _{2}\left(\sigma_{s}^{2}+\sum_{k=1}^{K} p_{k, s} g_{k, s}\right)-\sum_{s \in \mathcal{S}} \frac{B_{s}}{B} \log _{2}\left(\sigma_{s}^{2}\right) \\
& =\sum_{k=1}^{K} \sum_{s=1}^{S} \frac{B_{s}}{B} \log _{2}\left(1+\frac{p_{k, s} g_{k, s}}{\sigma_{s}^{2}+\sum_{j \in \mathcal{K} \backslash\{1, \ldots, k\}} p_{j, s} g_{j, s}}\right),
\end{aligned}
$$

we write (29) and (30) as follows,

$$
\operatorname{PoA}\left(\mathcal{G}_{(i)}\right)=\frac{\sum_{k=1}^{K} \sum_{s=1}^{S} \frac{B_{s}}{B} \log _{2}\left(1+\frac{p_{k, s}^{(\ddagger, i)} g_{k, s}}{\sigma_{s}^{2}+\sum_{j \in \mathcal{K} \backslash\{1, \ldots, k\}} p_{j, s}^{(\ddagger, i)} g_{j, s}}\right)}{\sum_{k=1}^{K} \sum_{s=1}^{S} \frac{B_{s}}{B} \log _{2}\left(1+\frac{p_{k, s}^{(\perp, i)} g_{k, s}}{\sigma_{s}^{2}+\sum_{j \in \mathcal{K} \backslash\{k\}} p_{j, s}^{(\perp, i)} g_{j, s}}\right)}
$$

and

$$
\operatorname{PoS}\left(\mathcal{G}_{(i)}\right)=\frac{\sum_{k=1}^{K} \sum_{s=1}^{S} \frac{B_{s}}{B} \log _{2}\left(1+\frac{p_{k, s}^{(\ddagger, i)} g_{k, s}}{\sigma_{s}^{2}+\sum_{j \in \mathcal{K} \backslash\{1, \ldots, k\}} p_{j, s}^{(\ddagger, i)} g_{j, s}}\right)}{\sum_{k=1}^{K} \sum_{s=1}^{S} \frac{B_{s}}{B} \log _{2}\left(1+\frac{p_{k, s}^{(\top, i)} g_{k, s}}{\sigma_{s}^{2}+\sum_{j \in \mathcal{K} \backslash\{k\}} p_{j, s}^{(\top, i)} g_{j, s}}\right)} .
$$

Finally, we highlight that, for all $k \in \mathcal{K}$ and for all $p \in$ $\mathcal{P}^{(a)}$ (and thus, for all $\boldsymbol{p} \in \mathcal{P}^{(b)}$ ), we have that,

$$
\begin{aligned}
& \sum_{s \in \mathcal{S}} \frac{B_{s}}{B} \log _{2}\left(1+\frac{p_{k, s} g_{k, s}}{\sigma_{s}^{2}+\sum_{j \in \mathcal{K} \backslash\{1, \ldots, k\}} p_{j, s} g_{j, s}}\right) \\
& \geqslant \sum_{s \in \mathcal{S}} \frac{B_{s}}{B} \log _{2}\left(1+\frac{p_{k, s} g_{k, s}}{\sigma_{s}^{2}+\sum_{j \in \mathcal{K} \backslash\{k\}} p_{j, s} g_{j, s}}\right) .
\end{aligned}
$$

Here, strict equality holds for all $k \in \mathcal{K}$, only when transmitters do not share the same channels with other transmitters. In the following, we use this reasoning to study the PoA and the PoS of both games $\mathcal{G}_{(a)}$ and $\mathcal{G}_{(b)}$.

\subsubsection{PoA and PoS in $\mathcal{G}_{(a)}$}

As shown in Theorem 1 , the set of NE of the game $\mathcal{G}_{(a)}$ is unitary. Thus, we have that,

$$
\boldsymbol{p}^{(\dagger, a)}=\boldsymbol{p}^{(\top, a)}=\boldsymbol{p}^{(\perp, a)},
$$

and thus,

$$
\operatorname{PoA}\left(\mathcal{G}_{(a)}\right)=\operatorname{PoS}\left(\mathcal{G}_{(a)}\right) .
$$

Following Proposition 2, it becomes clear that strict equality in (42) is observed at the equilibrium of the game $\mathcal{G}_{(a)}$, if and only if, transmitters use different channels to communicate with the receiver. In the case in which $K=2$ and $S=2$, according to Proposition 2, such condition is fulfilled when $\boldsymbol{g}=\left(g_{11}, g_{12}, g_{21}, g_{22}\right)$ satisfies either $\boldsymbol{g} \in \mathcal{A}_{1}$ or $g \in \mathcal{A}_{4}$. This observation leads us to the following lemma.

Lemma 5 (PoA and PoS of $\boldsymbol{G}_{(\boldsymbol{a})}$ ). Let $K=2, S=2$ and $\boldsymbol{g}=\left(g_{11}, g_{12}, g_{21}, g_{22}\right)$ satisfy at least one of the following conditions:

$$
\begin{aligned}
& \boldsymbol{g} \in\left\{\boldsymbol{g} \in \mathbb{R}_{+}^{4}: \frac{g_{11}}{g_{12}} \geqslant \frac{1+\mathrm{SNR} g_{11}}{1+\mathrm{SNR}_{22}}, \frac{g_{21}}{g_{22}} \leqslant \frac{1+\mathrm{SNR} g_{11}}{1+\mathrm{SNR} g_{22}}\right\} \text { or } \\
& \boldsymbol{g} \in\left\{\boldsymbol{g} \in \mathbb{R}_{+}^{4}: \frac{g_{11}}{g_{12}} \leqslant \frac{1+\mathrm{SNR} g_{21}}{1+\mathrm{SNR}_{12}}, \frac{g_{21}}{g_{22}} \geqslant \frac{1+\mathrm{SNR} g_{21}}{1+\mathrm{SNR} g_{12}}\right\} .
\end{aligned}
$$

Then,

$$
\operatorname{PoA}\left(\mathcal{G}_{(a)}\right)=\operatorname{PoS}\left(\mathcal{G}_{(a)}\right)=1 .
$$

Otherwise,

$$
\operatorname{PoA}\left(\mathcal{G}_{(a)}\right)=\operatorname{PoS}\left(\mathcal{G}_{(a)}\right)>1,
$$

with strict inequality.

From Lemma 5, the following corollaries follow by taking the limits SNR $\rightarrow 0$ and SNR $\rightarrow \infty$, respectively. 
Corollary 1 (PoA in the low SNR regime). Let $K=2$, $S=2$ and $\boldsymbol{g}=\left(g_{11}, g_{12}, g_{21}, g_{22}\right)$ satisfy at least one of the following conditions:

$$
\begin{array}{lll}
g_{11} \geqslant g_{12} & \text { and } \quad g_{21} \leqslant g_{22} \text { or } \\
g_{11} \leqslant g_{12} & \text { and } \quad g_{21} \geqslant g_{22} .
\end{array}
$$

Then, in the low SNR regime $(S N R \rightarrow 0)$,

$$
\operatorname{PoA}\left(\mathcal{G}_{(a)}\right)=\operatorname{PoS}\left(\mathcal{G}_{(a)}\right)=1,
$$

otherwise,

$$
\operatorname{PoA}\left(\mathcal{G}_{(a)}\right)=\operatorname{PoS}\left(\mathcal{G}_{(a)}\right)<1+\epsilon,
$$

with $\epsilon>0$ arbitrarily small.

Corollary 2 (PoA in the high SNR regime). Let $K=2$, $S=2$ and $\boldsymbol{g}=\left(g_{11}, g_{12}, g_{21}, g_{22}\right)$ satisfy at least one of the following conditions:

$$
\begin{aligned}
& g_{22} \geqslant g_{12} \text { and } g_{21} \leqslant g_{11} \text { or } \\
& g_{11} \leqslant g_{21} \text { and } g_{12} \geqslant g_{22} .
\end{aligned}
$$

Then, in the high SNR regime, we have that $(S N R \rightarrow \infty)$,

$$
\operatorname{PoA}\left(\mathcal{G}_{(a)}\right)=\operatorname{PoS}\left(\mathcal{G}_{(a)}\right)=1
$$

otherwise,

$$
\operatorname{PoA}\left(\mathcal{G}_{(a)}\right)=\operatorname{PoS}\left(\mathcal{G}_{(a)}\right) \rightarrow \infty .
$$

In a more general case, for instance $K>2$ and $S>2$, we state the following result:

Proposition 6 (PoA of the $\left.\boldsymbol{G}_{(\boldsymbol{a})}\right)$. Let $\boldsymbol{p}^{*} \in \mathcal{P}^{(a)}$ be the equilibrium of the game $\mathcal{G}_{(a)}$ and consider the following condition:

$$
\text { If } \exists(k, s) \in \mathcal{K} \times \mathcal{S}: p_{k, s}^{*}>0 \text {, then } \forall j \in \mathcal{K} \backslash\{k\}, p_{j, s}^{*}=0 \text {. }
$$

Then, if the condition above holds, we have that:

$$
\operatorname{PoA}\left(\mathcal{G}_{(a)}\right)=\operatorname{PoS}\left(\mathcal{G}_{(a)}\right)=1,
$$

otherwise, we have that:

$$
\begin{aligned}
\operatorname{PoA}\left(\mathcal{G}_{(a)}\right)=\operatorname{PoS}\left(\mathcal{G}_{(a)}\right) & >1 \text { and } \\
\lim _{\mathrm{SNR} \rightarrow \infty} \operatorname{PoA}\left(\mathcal{G}_{(a)}\right)=\lim _{\mathrm{SNR} \rightarrow \infty} \operatorname{PoS}\left(\mathcal{G}_{(a)}\right) & =\infty .
\end{aligned}
$$

Note that from Proposition 6, it can be concluded that in the case in which $K>S$, the PoA is strictly bigger than one, since the condition (57) does not hold. Thus, as long as $K>S$ there always exists a loss in performance due to the decentralization of the network. Nonetheless, depending on the channel realizations, in the case in which $K \leqslant S$, global optimal performance can be achieved at the equilibrium.

\subsubsection{PoA and PoS in $\mathcal{G}_{(b)}$}

As shown in Theorem 1, the set of NE of the game $\mathcal{G}_{(a)}$ is not necessarily unitary. Thus, we have that if the equilibrium of the game $\mathcal{G}_{(a)}$ is not unique, then $\boldsymbol{p}^{(\top, a)} \neq \boldsymbol{p}^{(\perp, a)}$ and the following holds:

$$
\begin{aligned}
\operatorname{PoA}\left(\mathcal{G}_{(b)}\right) & >1 \text { and } \\
\operatorname{PoS}\left(\mathcal{G}_{(b)}\right) & \geqslant 1,
\end{aligned}
$$

with strict inequality for any channel realization in (61). This is basically because two different NE action profiles generate the same sum utility with probability zero, when the channel gains are generated following a continuous distribution. Thus, the numerator is monotonically increasing while the denominator is monotonically decreasing in (40). On the contrary, in the case of the $\operatorname{PoS}(62)$, equality can be observed whenever $\boldsymbol{p}^{(\ddagger, b)}=$ $\boldsymbol{p}^{(\top, b)}$ and (33) holds with equality, which is the case when there exists an equilibrium where every transmitter uses a different channel from all the other transmitters.

Following the same arguments as in the case of the game $\mathcal{G}_{(a)}$, we state that a unitary PoA $(\operatorname{PoA}=1)$ is observed only when the set of NE is unitary and transmitters do not share the same channels to transmit. This condition according with Proposition 3 is observed in the game $\mathcal{G}_{(b)}$, with $K=2$ and $S=2$, when $\boldsymbol{g}=\left(g_{11}, g_{12}, g_{21}, g_{22}\right)$ satisfies that $\boldsymbol{g} \in\left\{\mathcal{B}_{1} \cup \mathcal{B}_{4}\right\} \backslash \boldsymbol{B}_{\mathbf{5}}$. This observation leads us to the following lemma.

Lemma 6 (PoA of the game $\mathcal{G}_{(b)}$ ). Let $K=2, S=2$ and $\boldsymbol{g}=\left(g_{11}, g_{12}, g_{21}, g_{22}\right)$ satisfy at least one of the following conditions:

$\boldsymbol{g} \in\left\{\boldsymbol{g} \in \mathbb{R}_{+}^{4}: \frac{g_{11}}{g_{12}} \geqslant 1+\mathrm{SNR} g_{21}, \frac{g_{21}}{g_{22}} \leqslant 1+\mathrm{SNR} g_{11}\right\}$

$$
\begin{gathered}
\boldsymbol{g} \in\left\{\boldsymbol{g} \in \mathbb{R}_{+}^{4}: \frac{1}{1+\mathrm{SNR} g_{22}} \leqslant \frac{g_{11}}{g_{12}} \leqslant 1+\mathrm{SNR} g_{21}, \frac{g_{21}}{g_{22}}\right. \\
\left.\leqslant \frac{1}{1+\mathrm{SNR} g_{12}}\right\}, \\
\boldsymbol{g} \in\left\{\boldsymbol{g} \in \mathbb{R}_{+}^{4}: \frac{g_{11}}{g_{12}} \leqslant \frac{1}{1+\mathrm{SNR} g_{22}}, \frac{1}{1+\mathrm{SNR} g_{12}}\right. \\
\left.\leqslant \frac{g_{21}}{g_{22}} \leqslant 1+\mathrm{SNR} g_{11}\right\} \text { or } \\
\boldsymbol{g} \in\left\{\boldsymbol{g} \in \mathbb{R}_{+}^{4}: \frac{g_{11}}{g_{12}} \leqslant 1+\mathrm{SNR} g_{21}, \frac{g_{21}}{g_{22}} \geqslant 1+\mathrm{SNR} g_{11}\right\} .
\end{gathered}
$$

Then,

$$
\operatorname{PoA}\left(\mathcal{G}_{(b)}\right)=1
$$


Otherwise,

$$
\operatorname{PoA}\left(\mathcal{G}_{(b)}\right)>1
$$

with strict inequality.

In the case of the PoS, we highlight that strict equality holds in (42) when transmitters use different channels to communicate with the receiver at the equilibrium. This condition is satisfied in the game $\mathcal{G}_{(b)}$, with $K=2$ and $S=2$, when $\boldsymbol{g}=\left(g_{11}, g_{12}, g_{21}, g_{22}\right)$ satisfies either $\boldsymbol{g} \in \mathcal{B}_{1}$ or $\boldsymbol{g} \in \mathcal{B}_{4}$. Thus, we write the following lemma.

Lemma 7 (PoS of the game $\left.\boldsymbol{G}_{(b)}\right)$. Let $K=2, S=2$ and $\boldsymbol{g}=\left(g_{11}, g_{12}, g_{21}, g_{22}\right)$ satisfy at least one of the following conditions:

$$
\boldsymbol{g} \in\left\{\boldsymbol{g} \in \mathbb{R}_{+}^{4}: \frac{g_{11}}{g_{12}} \geqslant \frac{1}{1+\mathrm{SNR} g_{22}}, \frac{g_{21}}{g_{22}} \leqslant 1+\mathrm{SNR}_{11}\right\} \text {, or }
$$

$$
\boldsymbol{g} \in\left\{\boldsymbol{g} \in \mathbb{R}_{+}^{4}: \frac{g_{11}}{g_{12}} \leqslant 1+\mathrm{SNR} g_{21}, \frac{g_{21}}{g_{22}} \geqslant \frac{1}{1+\mathrm{SNR} g_{12}}\right\} .
$$

Then,

$$
\operatorname{PoS}\left(\mathcal{G}_{(b)}\right)=1
$$

Otherwise,

$$
\operatorname{PoS}\left(\mathcal{G}_{(b)}\right)>1
$$

with strict inequality.

From Lemmas 6 and 7, the following corollaries follow by taking the limits SNR $\rightarrow 0$ and SNR $\rightarrow \infty$, respectively.

Corollary 3 (PoA and PoS in the low SNR regime). Let $K=2, S=2$ and $\boldsymbol{g}=\left(g_{11}, g_{12}, g_{21}, g_{22}\right)$ satisfy at least one of the following conditions:

$$
\begin{aligned}
& g_{11} \geqslant g_{12} \text { and } g_{21} \leqslant g_{22} \text { or } \\
& g_{11} \leqslant g_{12} \text { and } g_{21} \geqslant g_{22} .
\end{aligned}
$$

Then, in the low SNR regime $(S N R \rightarrow 0)$,

$$
\operatorname{PoA}\left(\mathcal{G}_{(b)}\right)=\operatorname{PoS}\left(\mathcal{G}_{(b)}\right)=1,
$$

otherwise,

$$
1<\operatorname{PoA}\left(\mathcal{G}_{(b)}\right)=\operatorname{PoS}\left(\mathcal{G}_{(b)}\right)<1+\epsilon,
$$

with $\epsilon>0$ arbitrarily small.
Corollary 4 (PoA and PoS in the high SNR regime). Let $K=2, S=2$. Then, in the high SNR regime $(S N R \rightarrow \infty)$, we have that

$$
\begin{aligned}
& \operatorname{PoA}\left(\mathcal{G}_{(b)}\right) \rightarrow \infty \\
& \operatorname{PoS}\left(\mathcal{G}_{(b)}\right) \rightarrow 1
\end{aligned}
$$

In Corollary 4, (77) follows from the fact that at high SNR, the game $\mathcal{G}_{(b)}$, with $K=2$ and $S=2$, always possesses two equilibria in pure strategies (Lemma 8). Thus, at the high SNR regime, the numerator of (29) arbitrarily increases while its denominator arbitrarily decreases. Similarly, at the high SNR regime both equilibria consist in action profiles where transmitters use different channels to communicate with the receiver. Thus, (42) holds with strict equality for the case of the NE with the highest NSE $\boldsymbol{p}^{\top}$, which justifies (78). In a more general case, for instance $K>2$ and $S>2$, we state the following result:

Proposition 7 (PoA of the $\left.\mathcal{G}_{(b)}\right)$. Let $\boldsymbol{p}^{*} \in \mathcal{P}^{(b)}$ be an equilibrium of the game $\mathcal{G}_{(b)}$ and consider the following conditions:

(i) $\boldsymbol{p}^{*}$ isunique

(ii)If $\exists(k, s) \in \mathcal{K} \times \mathcal{S}: p_{k, s}^{*}>0$, then $\forall j \in \mathcal{K} \backslash\{k\}, p_{j, s}^{*}=0$.

Then, if both conditions simultaneously hold, we have that:

$$
\operatorname{PoA}\left(\mathcal{G}_{(b)}\right)=1,
$$

otherwise, we have that:

$$
\begin{aligned}
\operatorname{PoA}\left(\mathcal{G}_{(b)}\right) & >1 \text { and } \\
\lim _{\mathrm{SNR} \rightarrow \infty} \operatorname{PoA}\left(\mathcal{G}_{(b)}\right) & =\infty .
\end{aligned}
$$

Proposition 8 (PoS of the $\left.\boldsymbol{G}_{(\boldsymbol{b})}\right)$. Let $\boldsymbol{p}^{*} \in \mathcal{P}^{(b)}$ be the equilibrium with the highest NSE of the game $\mathcal{G}_{(b)}$ and consider the following condition:

$$
\text { (i) } \begin{aligned}
\text { If } \exists(k, s) & \in \mathcal{K} \times \mathcal{S}: p_{k, s}^{*}>0, \\
\text { then } \forall j & \in \mathcal{K} \backslash\{k\}, p_{j, s}^{*}=0 .
\end{aligned}
$$

Then, if the condition above holds, we have that:

$$
\operatorname{PoS}\left(\mathcal{G}_{(b)}\right)=1 \text {, }
$$

otherwise, we have that:

$$
\begin{aligned}
\operatorname{PoS}\left(\mathcal{G}_{(b)}\right)>1 \text { and } \\
\lim _{\text {SNR } \rightarrow \infty} \operatorname{PoS}\left(\mathcal{G}_{(b)}\right) \rightarrow \infty .
\end{aligned}
$$

Note that from Proposition 7, it can be concluded that in the case in which $K>S$, the PoA and the PoS are both strictly bigger than one, since the condition (84) does 
not hold. Thus, as in the previous case, as long as $K>S$ there always exists a loss in performance due to the decentralization of the network. Nonetheless, depending on the channel realizations, in the case in which $K \leqslant S$, global optimal performance can be achieved at the equilibrium.

\section{Numerical examples}

In the previous sections, a mathematical argument has been provided to show that at the low and high SNR regimes, using a CS policy yields a higher or equal NSE than using a water-filling PA policy. A formal proof has been provided for the case of $K=2$ transmitters and $S=2$ channels. Moreover, we highlight that a CS policy is evidently simpler than a PA policy in terms of implementation. Unfortunately, providing a formal proof for an arbitrary number of transmitters $K$ and channels $S$ at a finite SNR becomes a hard task since it will require to calculate all the types of $\mathrm{NE}$ depending on the exact channel realizations. Hence, for the case of arbitrary parameters $K, S$, and SNR, we provide only numerical examples to give an insight of the general behavior. First, we evaluate the impact of the SNR for a network with a fixed number of transmitters and channels. Second, we evaluate the impact of the network load, i.e., the number of transmitters per channel for a given fixed SNR.

\subsection{Impact of the SNR $\frac{p_{\max }}{\sigma_{2}}$}

In Figure 4, we plot the NSE as a function of the average SNR of the transmitters. Here, it is shown that in fully loaded and over-loaded networks, i.e., $\eta=\frac{K}{S} \geqslant 1$, the gain in NSE obtained by using a discrete action set (game $\mathcal{G}_{(a)}$ ) increases with the SNR. Conversely, for weakly loaded networks $\eta<1$, limiting the transmitters to use a single channel appears to be suboptimal as the SNR increases. This is basically because using only one channel, necessarily implies letting some interference-free channels unused. Interestingly, at low SNR, the NSE observed in both games is the same, independently of the load of the system. In both cases, high SNR and low SNR regimes, the observed results are in line with Propositions 4 and 5 .

In Figure 5, we plot the probability of observing a specific number of NE in the game $\mathcal{G}_{(b)}$ for different values of SNR. This probability is calculated as the empirical frequency of the number of $\mathrm{NE}$ observed in $10^{6}$ realizations of the game $\mathcal{G}_{(a)}$ and $\mathcal{G}_{(b)}$. At each game realization, a new channel matrix realization $\boldsymbol{H}$ is considered. In the first case (Figure 5a), we consider $S=2$ and $K=3$, whereas in the second case (Figure 5b), $K=3$ and $S=3$. Note that from Proposition 1 , the maximum number of NE is 4 and 7, respectively. However, only 3 and $6 \mathrm{NE}$ are, respectively, observed in the simulations. This observation joints the discussion on the tightness of the bound presented in Section 3.3.2. Note that Proposition 1 relies only on the number of transmitters and available channels. A tighter bound can be obtained if the actual channel realizations are considered. However, this might be significantly tedious for networks with large number of transmitters.Moreover, note also that low SNR levels are associated with a unique NE (with high probability), whereas, high SNR levels are associated with multiple NE (with high probability). Note that this observation, at least for the case of $K=2$ and $S=2$, is inline with Propositions 4 and 5.

\subsection{Impact of the number of transmitters $(K)$}

In Figure 6, we plot the NSE as a function of the number of transmitters per channel, i.e., the system load $\eta=\frac{K}{S}$.

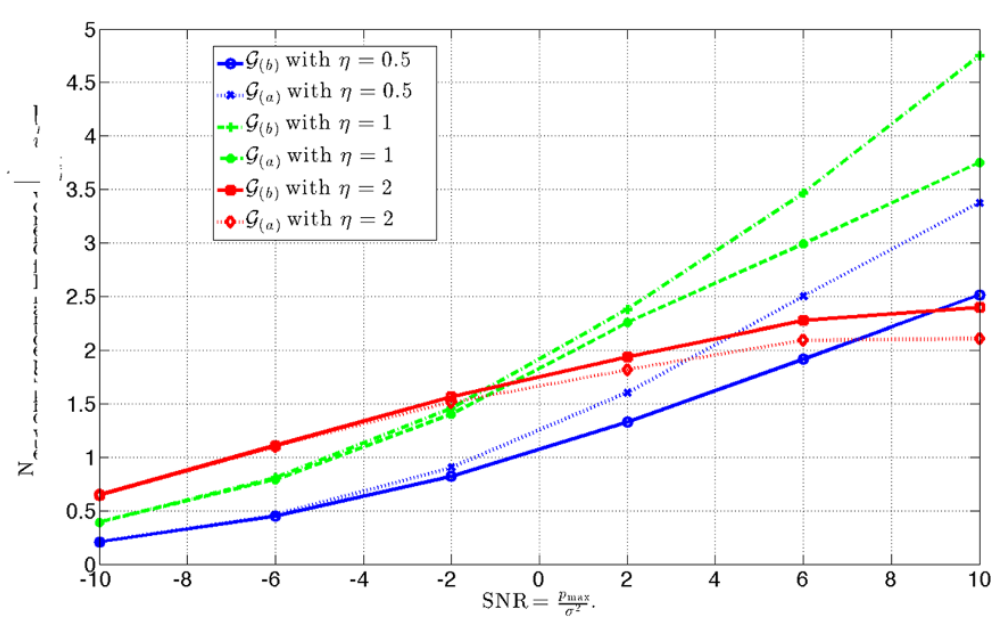

Figure 4 NSE as a function of the SNR $=\frac{p_{\max }}{\sigma^{2}}$ in dBs for the case of $\eta=\frac{K}{S} \in\left\{\frac{1}{2}, 1,2\right\}$, with $K=10$. 


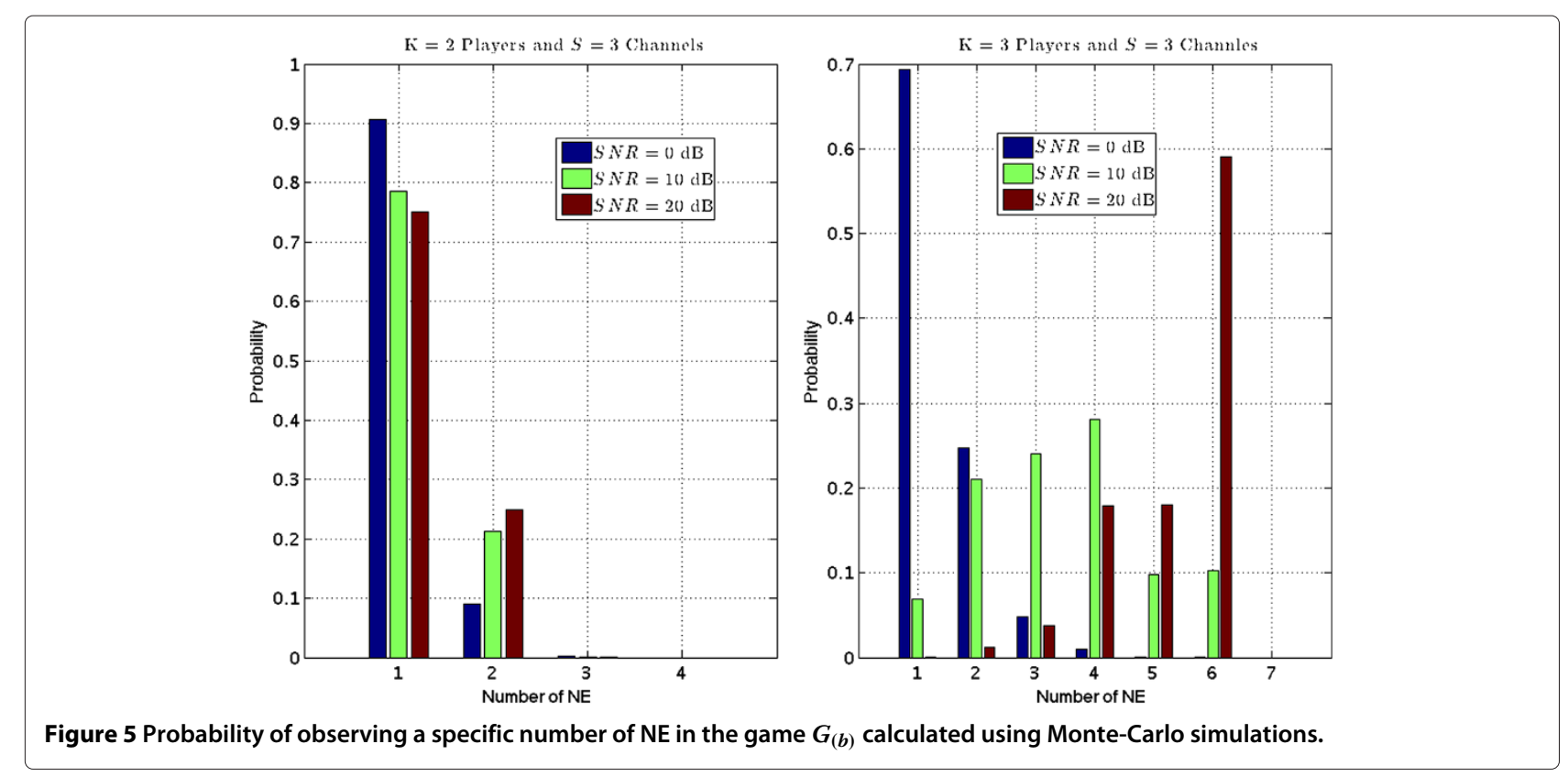

Therein, one can observe that for weakly loaded systems $\eta<1$, playing $\mathcal{G}_{(a)}$ always leads to higher NSE than playing $\mathcal{G}_{(b)}$. This is natural since restricting the transmitters to use only one channel implies not using other channels which are interference-free, as $S>K$. On the contrary, for fully loaded and over-loaded systems, the NSE of the game $\mathcal{G}_{(a)}$ is at least equal or better than that of the game $\mathcal{G}_{(b)}$. Indeed, the fact that for high system loads $\eta>2$, the NSE obtained by playing the game $\mathcal{G}_{(a)}$ and $\mathcal{G}_{(b)}$ become identical stems from the fact that under this condition the system becomes dominated by the interference.
In Figure 7, we plot the PoA (29) of both games $\mathcal{G}_{(a)}$ and $\mathcal{G}_{(b)}$ as a function of the number of transmitters for several SNR values. First, we highlight the fact that as shown in our theoretical analysis, optimal performance $\left(\operatorname{PoA}\left(\mathcal{G}_{(b)}\right)=1\right)$ is achieved in the case of low density networks $\eta \leqslant 1$ using CS. Note also that in the case of the PA game $\operatorname{PoA}\left(\mathcal{G}_{(a)}\right)>1$ independently of the network load, which implies that there always exists a loss of performance when the network is decentralized and the transmitters are allowed to use several channels simultaneously.

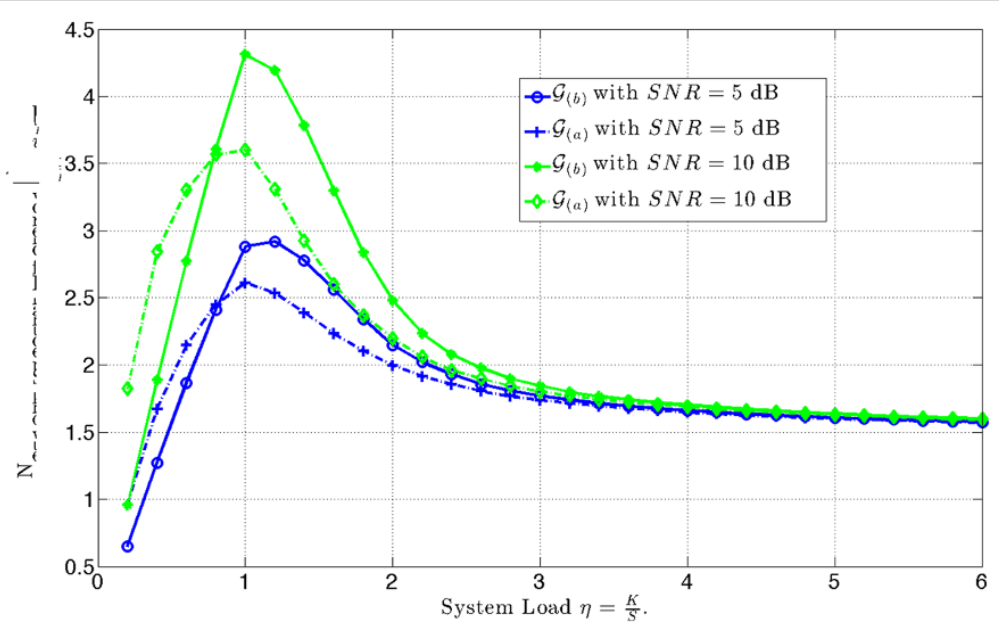

Figure 6 NSE as a function of the system load $\eta=\frac{K}{S}$ for different SNR $=\frac{p_{\max }}{\sigma^{2}}$ levels in dB. 


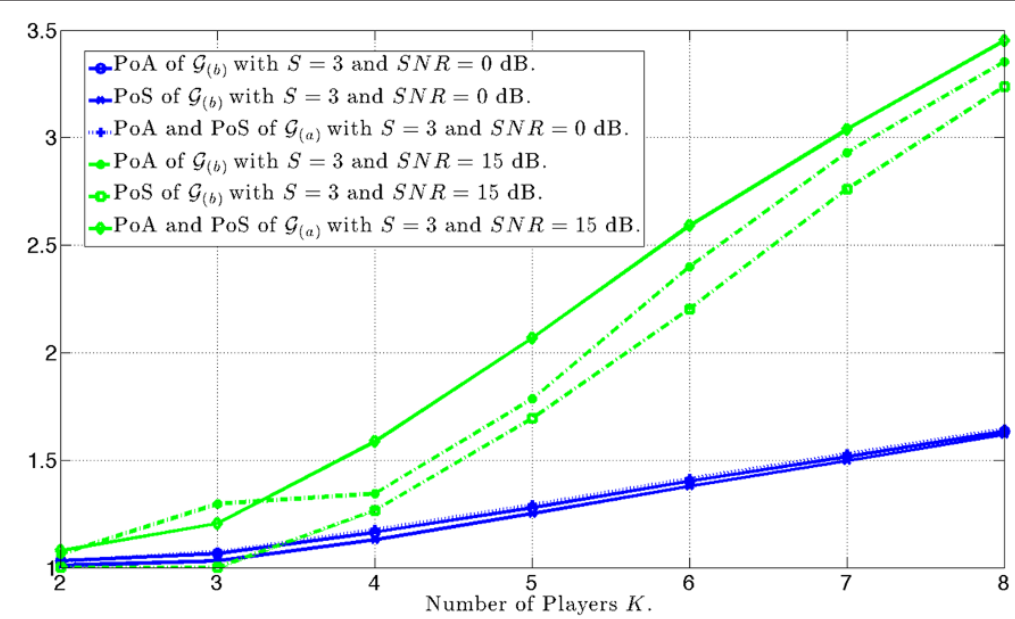

Figure 7 PoA calculated using Monte Carlo simulations and using Equation (29) for a network with $K=10$ players, $\frac{B_{s}}{B}=\frac{1}{S}$, for all $s \in S$ and SNR $=10 \log _{10}\left(\frac{p_{\max }}{N_{0} B}\right) \in\{0,15\} \mathrm{dB}$.

Finally, in Figure 8, we show the fractions $x_{s}$ of transmitters using channel $s$, with $s \in \mathcal{S}$, obtained by Monte Carlo simulations and using (26) for a large network with an asymptotic ratio of players per channel equivalent to $\eta=10$. Therein, it becomes clear that (26) is a precise estimation of the outcome of the games $\mathcal{G}_{(a)}$ and $\mathcal{G}_{(b)}$ in the regime of large number of players.

\section{Conclusions}

In this article, it is shown to what extent the equilibrium analysis of the decentralized parallel MAC differs from those conducted for other channels like Gaussian MIMO interference channels and fast fading MIMO MAC. In particular, the special structure of parallel MAC and the assumption of SUD at the receiver leads to the potential game property. The CS game was merely introduced in the literature but not investigated in details as it is in this article. In particular, a graph-theoretic interpretation is used to characterize the number of NE. In the case where the number of transmitters is large, the fraction between pure $\mathrm{NE}$ and the total number of action profiles is relatively small, which makes both the analysis and the achievability

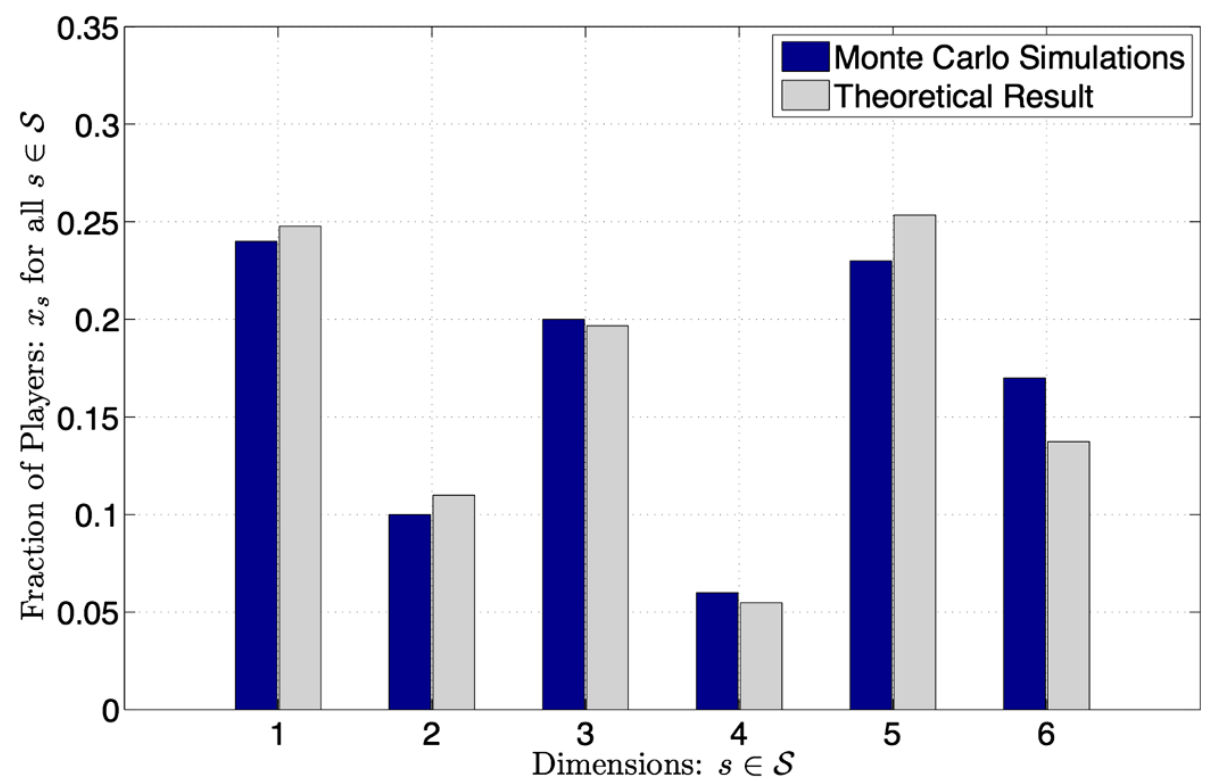

Figure 8 Fraction of players transmitting over channel $s$, with $s \in S$, calculated using Monte Carlo simulations and using Equation (26) for a network with $S=6$ channels, $K=60$ players, with $b=\left(b_{s}\right)_{\forall s \in \mathcal{S}}=(0.25,0.11,0.20,0.05,0.25,0.14)$, and SNR $=10 \log _{10}\left(\frac{p_{\max }}{N_{0} B}\right)=10 \mathrm{~dB}$. 
of the NE a challenging task. Now, from a design point of view, we provide theoretical results and numerical examples to show that a fully loaded network, when transmitters use only one channel, can be more efficient than its counterpart when all the channels can be exploited by the transmitters. Although all of these results are encouraging about the relevance of game-theoretic analyses of PA problems, important practical issues have deliberately been ignored. For example, the impact of channel estimation is not assessed at all. Also, it is important to conduct a detailed analysis on the signaling cost involved by all the PA algorithms arising from this game formulations to learn NE.

\section{Appendix}

\section{Proof of Proposition 1}

In this appendix, we provide a proof of Proposition 1, which establishes an upper bound for the number of NE of the game $\mathcal{G}_{(b)}$. Here, we exploit some basic tools from graph theory. Let us index the elements of the action set $\mathcal{P}^{(b)}$ in any given order using the index $n \in \mathcal{I}=$ $\left\{1, \ldots, S^{K}\right\}$. Denote by $\boldsymbol{p}^{(n)}$ the $n$th element of the action set $\mathcal{P}^{(b)}$. We write each vector $\boldsymbol{p}^{(n)}$ with $n \in \mathcal{I}$, as $\boldsymbol{p}^{(n)}=$ $\left(\boldsymbol{p}_{1}^{(n)}, \ldots, \boldsymbol{p}_{K}^{(n)}\right)$, where for all $j \in \mathcal{K}, \boldsymbol{p}_{j}^{(n)} \in \mathcal{P}_{j}^{(b)}$. Consider that each action profile $\boldsymbol{p}^{(n)}$ is associated with a vertex $v_{n}$ in a given non-directed graph $G$. Each vertex $v_{n}$ is adjacent to the $K(S-1)$ vertices associated with the action profiles resulting when only one player deviates from the action profile $\boldsymbol{p}^{(n)}$, i.e., if two vertices $v_{n}$ and $v_{m}$, with $(n, m) \in \mathcal{I}^{2}$ and $n \neq m$, are adjacent, then there exists one and only one $k \in \mathcal{K}$, such that

$$
\forall j \in \mathcal{K} \backslash\{k\}, \boldsymbol{p}_{j}^{(n)}=\boldsymbol{p}_{j}^{(m)} \text {, and } \boldsymbol{p}_{k}^{(n)} \neq \boldsymbol{p}_{k}^{(m)} \text {. }
$$

More precisely, the graph $G$ can be defined by the pair $G=(\mathcal{V}, \boldsymbol{A})$, where the set $\mathcal{V}=\left\{v_{1}, \ldots, v_{S^{K}}\right\}$ (nodes) represents the $S^{K}$ possible actions profiles of the game and $\boldsymbol{A}$ (edges) is a symmetric matrix (adjacency matrix of $G$ ) with dimensions $S^{K} \times S^{K}$ and entries defined as follows $\forall(n, m) \in \mathcal{I}^{2}$ and $n \neq m$,

$$
a_{n, m}=a_{m, n}=\left\{\begin{array}{l}
1 \text { if } n \in \mathcal{V}_{m} \\
0 \text { otherwise }
\end{array}\right.
$$

and $a_{n, n}=0$ for all $n \in \mathcal{I}$, where the set $\mathcal{V}_{n}$ is the set of indices of the adjacent vertices of vertex $v_{n}$. In the following, we use the concept of distance between two vertices of the graph $G$. We define this concept using our notation:

Definition 4 (Shortest Path). The distance (shortest path) between vertices $v_{n}$ and $v_{m}$, with $(n, m) \in \mathcal{I}^{2}$ in a given non-directed graph $G=(\mathcal{V}, A)$, denoted by $d_{n, m}(G) \in \mathbb{N}$ is:

$$
d_{n, m}(G)=d_{m, n}(G)=\sum_{k=1}^{K} \mathbb{1}_{\left\{\boldsymbol{p}_{k}^{(n)} \neq \boldsymbol{p}_{k}^{(m)}\right\}} .
$$

Here, for any pair of action profiles $\boldsymbol{p}^{(n)}$ and $\boldsymbol{p}^{(m)}$, with $(n, m) \in \mathcal{I}^{2}$ and $n \neq m$, we have that $\phi\left(\boldsymbol{p}^{(n)}\right) \neq \phi\left(\boldsymbol{p}^{(m)}\right)$ with probability one. This is because channel gains are random variables drawn from continuous probability distributions and thus, $\operatorname{Pr}\left(\phi\left(\boldsymbol{p}^{(n)}\right)=\phi\left(\boldsymbol{p}^{(m)}\right) \mid n \neq m\right)=0$. Hence, following Definition 1 , one can state that if the action profile $\boldsymbol{p}^{(n)}$, with $n \in \mathcal{I}$, is an NE of the game $\mathcal{G}_{(b)}$, then, it follows that

$$
\forall m \in \mathcal{V}_{(n)}, \quad \phi\left(\boldsymbol{p}^{(n)}\right)>\phi\left(\boldsymbol{p}^{(m)}\right),
$$

and thus, with probability one, none on the action profiles in $\mathcal{V}_{(n)}$ are NE. By iteratively following this procedure over the remaining set of action profiles $\mathcal{I} \backslash\left\{n \cup \mathcal{V}_{(n)}\right\}$, some action profiles can be eliminated and only those that are potentially NE are left over.

Thus, given that the action profile $\boldsymbol{p}^{(n)}$ is an NE, the action profile $\boldsymbol{p}^{(m)}$, with $n \neq m$, can be an NE candidate, if it is (at least) at distance two of $\boldsymbol{p}^{(n)}$ and any other NE candidate, i.e., $d_{n, m}(G)=d_{m, n}(G) \in\{2,4, \ldots, \hat{K}\}$. An action profile at distance $\ell \in\{2,4, \ldots, \hat{K}\}$ from $\boldsymbol{p}^{(n)}$, is a vector where $\ell$ players have simultaneously deviated from $\boldsymbol{p}^{(n)}$. Hence, for each $\ell$-tuple of players, there always exists $S-1$ action profiles at distance $\ell$ from $\boldsymbol{p}^{(n)}$ and at distance 2 from each other. Thus, considering the initial NE action profile $\boldsymbol{p}^{(n)}$, there might exist at most

$$
L \leqslant 1+\sum_{i \in\{2,4, \ldots, \hat{K}\}}\left(\begin{array}{c}
K \\
i
\end{array}\right)(S-1)
$$

NE candidates. This establishes an upper bound for $L$ and completes the proof.

\section{Proof of Proposition 2}

In this appendix, we provide a proof for the Proposition 2. The proof is separated in two steps. First, we show that a PA vector $\boldsymbol{p}=\left(\boldsymbol{p}_{1}, \boldsymbol{p}_{2}\right) \in \mathcal{P}^{(a)}$ of the form

$$
\boldsymbol{p}_{1}=\left(p_{11}, p_{\max }-p_{11}\right) \text { and } \boldsymbol{p}_{2}=\left(p_{\max }-p_{22}, p_{22}\right),
$$

is not an NE of the game $\mathcal{G}_{(a)}$, when $\left.p_{11} \in\right] 0, p_{\max }[$ and $\left.p_{22} \in\right] 0, p_{\max }[$. Second, we show that if $\boldsymbol{p}$ is an NE, then, $\boldsymbol{p}$ is unique and satisfies that, $\boldsymbol{p} \in \mathcal{P}^{\dagger}$, where

$$
\begin{aligned}
\mathcal{P}^{\dagger}= & \mathcal{P}^{(a)} \backslash\left\{\boldsymbol{p}=\left(p_{11}, p_{\max }-p_{11}, p_{\max }-p_{22}, p_{22}\right)\right. \\
& \left.\in \mathbb{R}_{+}^{4}: p_{11} \in\right] 0, p_{\max }\left[\text { and } p_{22} \in\right] 0, p_{\max }[\} .
\end{aligned}
$$

In the following, we use the notation $-c$ to denote the element other than $c$ in the binary set $\mathcal{C}$. 
First step: Assume that the action profile $\boldsymbol{p}=\left(\boldsymbol{p}_{1}, \boldsymbol{p}_{2}\right)$, with $\boldsymbol{p}_{1}=\left(p_{11}, p_{12}\right)$ and $\boldsymbol{p}_{2}=\left(p_{21}, p_{22}\right)$ is an NE of the game $\mathcal{G}_{(a)}$, and assume that for all $(k, s) \in \mathcal{K} \times \mathcal{S}, p_{k, s}>$ 0 , with strict inequality. Then, from the best response correspondence, it holds that $\forall(k, s) \in \mathcal{K} \times \mathcal{S}$,

$$
p_{k, s}^{\dagger}=\left[\frac{1}{\beta_{k}}-\frac{\sigma^{2}+g_{-k, s} p_{-k, s}^{\dagger}}{g_{k, s}}\right]^{+},
$$

with $\beta_{k}$ a Lagrangian multiplier chosen to satisfy (2). Then, from (92), it can be implied that $\forall k \in \mathcal{K}$,

$$
\begin{aligned}
p_{k, s} & =\frac{1}{\beta_{k}}-\frac{\sigma^{2}+p_{-k, s} g_{-k, s}}{g_{k, s}}>0 \text { and } \\
p_{k,-s} & =\frac{1}{\beta_{k}}-\frac{\sigma^{2}+p_{-k,-s} g_{-k,-s}}{g_{k,-s}}>0 .
\end{aligned}
$$

Then, from the fact that $\forall k \in \mathcal{K}, p_{k, s}+p_{k,-s}=p_{\text {max }}$, we have that,

$$
\begin{aligned}
& p_{k, k}=\frac{1}{2}\left(p_{\max }-\frac{\sigma^{2}+g_{-k, k}\left(p_{\max }-p_{-k,-k}\right)}{g_{k, k}} \frac{\sigma^{2}+g_{-k,-k} p_{-k,-k}}{g_{k,-k}}\right) \\
& p_{k,-k}=p_{\max }-p_{k, k} .
\end{aligned}
$$

Using a matrix notation, the system of equations (95) can be written in terms of $p_{11}$ and $p_{22}$ as follows:

$$
C\left(\begin{array}{l}
p_{11} \\
p_{22}
\end{array}\right)=A
$$

where the matrix $C$ is

$$
\boldsymbol{C}=\left(\begin{array}{cc}
2 g_{11} g_{12} & -\left(g_{22} g_{11}+g_{21} g_{12}\right) \\
-\left(g_{22} g_{11}+g_{21} g_{12}\right) & 2 g_{22} g_{21}
\end{array}\right)
$$

and, the vector $A$ is

$$
\boldsymbol{A}=\left(\begin{array}{l}
p_{\max } g_{12}\left(g_{11}-g_{21}\right)+\sigma^{2}\left(g_{11}-g_{12}\right) \\
p_{\max } g_{21}\left(g_{22}-g_{12}\right)+\sigma^{2}\left(g_{22}-g_{21}\right)
\end{array}\right) .
$$

Note that the system of equations (96) has a unique solution as long as the set of channels $\left\{g_{11}, g_{12}, g_{21}, g_{22}\right\}$ satisfies the condition $g_{12} g_{21}-g_{11} g_{22} \neq 0$. Let us continue the analysis under the assumption that, $g_{12} g_{21}-g_{11} g_{22} \neq 0$ (the case where $g_{12} g_{21}-g_{11} g_{22}=0$ is treated later). Then, the unique solution of (96) is $\forall k \in \mathcal{K}$,

$$
\begin{aligned}
p_{k, k} & =\frac{p_{\max } g_{-k, k}\left(g_{k,-k}+g_{-k,-k}\right)}{g_{12} g_{21}-g_{11} g_{22}} \\
& +\frac{\sigma^{2}\left(g_{-k, k}+g_{-k,-k}\right)}{g_{12} g_{21}-g_{11} g_{22}}, \\
p_{k,-k} & =p_{\max }-p_{k, k} .
\end{aligned}
$$

Note that if $g_{12} g_{21}-g_{11} g_{22}<0$, then $\forall k \in \mathcal{K}, p_{k, k}<0$, and, if $g_{12} g_{21}-g_{11} g_{22}>0$, then $\forall k \in \mathcal{K}, p_{k, k}>p_{\max }$, which contradicts the initial power constraints (2). Hence, any vector $\boldsymbol{p}=\left(\boldsymbol{p}_{1}, \boldsymbol{p}_{2}\right)$, with $\boldsymbol{p}_{1}=\left(p_{11}, p_{\max }-p_{11}\right)$ and $\boldsymbol{p}_{2}=\left(p_{\max }-p_{22}, p_{22}\right)$, such that $\forall(k, s) \in \mathcal{K} \times \mathcal{S}, 0<$ $p_{k, s}<p_{\max }$ is not an NE for the game $\mathcal{G}_{(a)}$ when $g_{12} g_{21}-$ $g_{11} g_{22} \neq 0$. Assume now that $g_{12} g_{21}-g_{11} g_{22}=0$, and let $\alpha=\frac{g_{21}}{g_{11}}=\frac{g_{22}}{g_{12}}$. Then, the PA vector in (95) can be written as follows, for $k=1$

$$
\left\{\begin{array}{l}
p_{11}=\alpha p_{22}+\frac{1}{2}\left(p_{\max }(1-\alpha)+\sigma^{2}\left(\frac{1}{g_{12}}-\frac{1}{g_{11}}\right)\right) \\
p_{12}=p_{\max }-p_{11} .
\end{array}\right.
$$

and, for $k=2$,

$$
\left\{\begin{array}{l}
p_{22}=\frac{1}{2}\left(p_{\max }\left(1+\frac{1}{\alpha}\right)+\sigma^{2}\left(\frac{1}{g_{21}}-\frac{1}{g_{22}}\right)\right)+\frac{1}{\alpha} p_{11} \\
p_{21}=p_{\max }-p_{22}
\end{array}\right.
$$

Note that the first equations in both (98) and (99) are identical. Thus, we focus only on the first equation in (98). This implies that any PA vector, $\boldsymbol{p}=\left(\boldsymbol{p}_{1}, \boldsymbol{p}_{2}\right)$, with $\boldsymbol{p}_{1}=$ $\left(p_{11}, p_{\max }-p_{11}\right) \in \mathcal{P}_{1}^{(a)}$ and $\boldsymbol{p}_{2}=\left(p_{\max }-p_{22}, p_{22}\right) \in$ $\mathcal{P}_{2}^{(a)}$ satisfying the condition

$$
p_{11}=\frac{1}{2}\left(p_{\max }(1-\alpha)+\sigma^{2}\left(\frac{1}{g_{12}}-\frac{1}{g_{11}}\right)\right)+\alpha p_{22}
$$

is an NE of the game $\mathcal{G}_{(a)}$ when $g_{12} g_{21}-g_{11} g_{22}=0$ as long as $\forall(k, s) \in \mathcal{K} \times \mathcal{S}, 0<p_{k, s}<p_{\max }$. For satisfying the latter conditions, it suffices to ensure that: $0<p_{11}<p_{\max }$ when $p_{22}=0$ and $p_{22}=p_{\max }$. Solving these inequalities leads to the following conditions over the channels:

(i) $p_{11}>0$, when $p_{22}=0$, if

$$
\frac{1+\frac{p_{\max }}{\sigma^{2}} g_{21}}{1+\frac{p_{\max }}{\sigma^{2}} g_{12}}<\frac{g_{11}}{g_{12}}
$$

(ii) $p_{11}>0$, when $p_{22}=p_{\max }$, if

$$
\frac{1}{1+\frac{p_{\max }}{\sigma^{2}}\left(g_{12}+g_{22}\right)}<\frac{g_{11}}{g_{12}} \text {. }
$$

(iii) $p_{11}<p_{\max }$, when $p_{22}=0$, if

$$
\frac{g_{11}}{g_{12}}<1+\frac{p_{\max }}{\sigma^{2}}\left(g_{11}+g_{21}\right)
$$

(iv) $p_{11}<p_{\max }$, when $p_{22}=p_{\max }$, if

$$
\frac{g_{11}}{g_{12}}<1+\frac{p_{\max }}{\sigma^{2}}\left(g_{11}+g_{21}\right)
$$

Finally, we obtain that if the vector of channels $g=$ $\left(g_{11}, g_{12}, g_{21}, g_{22}\right)$ satisfies that

$$
\begin{gathered}
\min \left(\frac{1}{1+\frac{p_{\max }}{\sigma^{2}}\left(g_{12}+g_{22}\right)}, \frac{1+\frac{p_{\max }}{\sigma^{2}} g_{21}}{1+\frac{p_{\max }}{\sigma^{2}} g_{12}}\right)<\alpha \text { and } \\
\max \left(1+\frac{p_{\max }}{\sigma^{2}}\left(g_{11}+g_{21}\right), \frac{1+\frac{p_{\max }}{\sigma^{2}} g_{11}}{1+\frac{p_{\max }}{\sigma^{2}} g_{22}}\right)>\alpha,
\end{gathered}
$$


that is,

$$
\frac{1}{1+\frac{p_{\max }}{\sigma^{2}}\left(g_{12}+g_{22}\right)}<\alpha<1+\frac{p_{\max }}{\sigma^{2}}\left(g_{11}+g_{21}\right),
$$

then any vector $\boldsymbol{p}=\left(\boldsymbol{p}_{1}, \boldsymbol{p}_{2}\right)$, with $\boldsymbol{p}_{1}=\left(p_{11}, p_{\max }-\right.$ $\left.p_{11}\right) \in \mathcal{P}_{1}^{(a)}$ and $\boldsymbol{p}_{2}=\left(p_{\max }-p_{22}, p_{22}\right) \in \mathcal{P}_{2}^{(a)}$ satisfying the condition (100) is an NE of the game $\mathcal{G}_{(a)}$. Note that infinitely many PA vectors might satisfy (100), which implies infinitely many NE. However, since the channels are realizations of random variables drawn from a continuous distribution, the probability of observing a realization such that $g_{12} g_{21}-g_{11} g_{22}=0$ is zero. Thus, with probability one, any vector $\boldsymbol{p}=\left(\boldsymbol{p}_{1}, \boldsymbol{p}_{2}\right)$, with $\boldsymbol{p}_{1}=$ $\left(p_{11}, p_{\max }-p_{11}\right)$ and $\boldsymbol{p}_{2}=\left(p_{\max }-p_{22}, p_{22}\right)$, such that $\forall(k, s) \in \mathcal{K} \times \mathcal{S}, 0<p_{k, s}<p_{\max }$ is not an NE for the game $\mathcal{G}_{(a)}$.

Second step: Consider that $\boldsymbol{p}^{\dagger}=\left(\boldsymbol{p}_{1}^{\dagger}, \boldsymbol{p}_{2}^{\dagger}\right) \in \mathcal{P}^{(a)}$ is an NE. Then, it must follow that $\boldsymbol{p}^{\dagger} \in \mathcal{P}^{\dagger}$, where,

$$
\begin{aligned}
\mathcal{P}^{\dagger}= & \mathcal{P} \backslash\left\{\boldsymbol{p}=\left(p_{11}, p_{\max }-p_{11}, p_{\max }-p_{22}, p_{22}\right)\right. \\
& \left.\times \in \mathbb{R}_{+}^{4}: p_{11} \in\right] 0, p_{\max }\left[\text { and } p_{22} \in\right] 0, p_{\max }[\} \\
= & \bigcup_{n=1}^{8} \mathcal{P}_{i}^{\dagger},
\end{aligned}
$$

where the sets $\mathcal{P}_{n}^{\dagger} \subset \mathcal{P}^{(a)}$, for all $n \in\{1, \ldots, 8\}$ are described as follows. The singletons $\mathcal{P}_{1}^{\dagger}=\{\boldsymbol{p}=$ $\left.\left(p_{\max }, 0,0, p_{\max }\right)\right\}, \mathcal{P}_{2}^{\dagger}=\left\{\boldsymbol{p}=\left(p_{\max }, 0, p_{\max }, 0\right)\right\}, \mathcal{P}_{3}^{\dagger}=$ $\left\{\boldsymbol{p}=\left(0, p_{\max }, 0, p_{\max }\right)\right\}, \mathcal{P}_{4}^{\dagger}=\left\{\boldsymbol{p}=\left(0, p_{\max }, p_{\max }, 0\right)\right\}$ and the convex non-closed sets,

$$
\begin{gathered}
\mathcal{P}_{5}^{\dagger}=\left\{\boldsymbol{p}=\left(p_{11}, p_{\max }-p_{11}, p_{\max }-p_{22}, p_{22}\right) \in \mathbb{R}_{+}^{4}:\right. \\
\left.p_{11}=p_{\max }, \text { and } p_{22} \in\right] 0, p_{\max }[\}, \\
\mathcal{P}_{6}^{\dagger}=\left\{\boldsymbol{p}=\left(p_{11}, p_{\max }-p_{11}, p_{\max }-p_{22}, p_{22}\right) \in \mathbb{R}_{+}^{4}:\right. \\
\left.p_{11} \in\right] 0, p_{\max }\left[\text { and } p_{22}=p_{\max }\right\}, \\
\mathcal{P}_{7}^{\dagger}=\left\{\boldsymbol{p}=\left(p_{11}, p_{\max }-p_{11}, p_{\max }-p_{22}, p_{22}\right) \in \mathbb{R}_{+}^{4}:\right. \\
\\
\left.p_{11} \in\right] 0, p_{\max }\left[\text { and } p_{22}=0\right\}, \\
\mathcal{P}_{8}^{\dagger}=\left\{\boldsymbol{p}=\left(p_{11}, p_{\max }-p_{11}, p_{\max }-p_{22}, p_{22}\right) \in \mathbb{R}_{+}^{4}:\right. \\
\left.p_{11}=0, \text { and } p_{22} \in\right] 0, p_{\max }[\} .
\end{gathered}
$$

In the following, we identify the conditions over the channel vector $\boldsymbol{g}=\left(g_{11}, g_{12}, g_{21}, g_{22}\right)$ such that each $\boldsymbol{p}^{\dagger} \in$ $\mathcal{P}_{n}^{\dagger}$, with $n \in\{1, \ldots, 8\}$ is an NE.
Assume that $\boldsymbol{p}^{\dagger} \in \mathcal{P}_{8}^{\dagger}$, i.e., $\boldsymbol{p}_{1}^{\dagger}=\left(0, p_{\max }\right)$ and $\boldsymbol{p}_{2}^{\dagger}=$ $\left(p_{\max }-p_{22}^{\dagger}, p_{22}^{\dagger}\right)$, with $\left.p_{22}^{\dagger} \in\right] 0, p_{\max }[$. Then, from (92) with $k=2$, we have that:

$$
\begin{aligned}
& p_{21}^{\dagger}=\frac{1}{\beta_{2}}-\frac{\sigma^{2}}{g_{21}}>0 \text { and } \\
& p_{22}^{\dagger}=\frac{1}{\beta_{2}}-\frac{\sigma^{2}+g_{12} p_{\max }}{g_{22}}>0 .
\end{aligned}
$$

Then, since $p_{21}^{\dagger}+p_{22}^{\dagger}=p_{\max }$, we have that $\frac{1}{\beta_{2}}=$ $\frac{1}{2}\left(p_{\max }+\frac{\sigma^{2}+p_{\max } g_{12}}{g_{22}}+\frac{\sigma^{2}}{g_{21}}\right)$, and thus,

$$
p_{22}^{\dagger}=\frac{1}{2}\left(p_{\max }-\frac{\sigma^{2}+g_{12} p_{\max }}{g_{22}}+\frac{\sigma^{2}}{g_{21}}\right),
$$

where, it must satisfy that $0<p_{22}^{\dagger}<p_{\text {max }}$. The inequality $p_{22}^{\dagger}>0$, holds only if $\frac{g_{21}}{g_{22}}<\frac{1+\frac{p_{\max } g_{21}}{\sigma^{2}}}{1+\frac{p_{\max } \sigma_{12}}{\sigma^{2}}}$, whereas the inequality $p_{22}^{\dagger}<p_{\text {max }}$ holds only if $\frac{g_{21}}{g_{22}}>\frac{1}{1+\operatorname{SNR}\left(g_{12}+g_{22}\right)}$. Similarly, from (92) with $k=1$, we have that given $p_{22}^{\dagger}$, in order to obtain $p_{11}^{\dagger}=0$ and $p_{12}^{\dagger}=p_{\max }$, it must hold that

$$
\begin{aligned}
& p_{11}=\frac{1}{\beta_{1}}-\frac{\sigma^{2}+g_{21}\left(p_{\max }-p_{22}^{\dagger}\right)}{g_{11}} \leqslant 0 \text { and } \\
& p_{12}=\frac{1}{\beta_{1}}-\frac{\sigma^{2}+g_{22} p_{22}^{\dagger}}{g_{12}} \geqslant p_{\max } .
\end{aligned}
$$

Hence, by doing $p_{12}-p_{11}$ in (109), we obtain that:

$$
\frac{\sigma^{2}+g_{21}\left(p_{\max }-p_{22}^{\dagger}\right)}{g_{11}}-\frac{\sigma^{2}+g_{22} p_{22}^{\dagger}}{g_{12}} \leqslant p_{\max } .
$$

Then, by replacing (108) in (110), we obtain that the condition (109) are satisfied only if the channels satisfy that:

$$
\frac{g_{11}}{g_{12}} \leqslant \frac{g_{21}}{g_{22}}
$$

Hence, we can conclude that whenever the vector $\boldsymbol{g}=\left(g_{11}, g_{12}, g_{21}, g_{22}\right) \in \mathcal{A}_{8}$, the NE is of the form $\left(p_{11}, p_{\max }-p_{11}, p_{\max }-p_{22}, p_{22}\right)$, with $p_{11}=0$ and $p_{22}=$ $\frac{1}{2}\left(p_{\max }-\frac{\sigma^{2}+g_{12} p_{\max }}{g_{22}}+\frac{\sigma^{2}}{g_{21}}\right)$. Now, assuming that $\boldsymbol{p}^{\dagger} \in$ $\mathcal{P}_{n}^{\dagger}$, with $n \in\{1, \ldots, 7\}$, leads to the conditions of the other types of NE, i.e., the corresponding sets $\mathcal{A}_{n}$, such that whenever $\boldsymbol{g} \in \mathcal{A}_{n}$ then $\boldsymbol{p}^{\dagger} \in \mathcal{P}_{n}^{\dagger}$. It is important to note that, for any particular vector $\boldsymbol{g}=\left(g_{11}, g_{12}, g_{21}, g_{22}\right) \in \mathcal{R}^{4}$, there exists, with probability one, only one set $\mathcal{A}_{n}$ which satisfies that $g \in \mathcal{A}_{n}$. This is basically because for all $(n, m) \in\{1, \ldots, 4\}^{2}$, with $n \neq m$, it follows that $\mathcal{A}_{n} \cap \mathcal{A}_{m}=$ $\emptyset$. Now, for all $(n, m) \in\{5, \ldots, 8\}^{2}$, with $n \neq m$, it follows that $\mathcal{A}_{n} \cap \mathcal{A}_{m} \subset\left\{\boldsymbol{g}=\left(g_{11}, g_{12}, g_{21}, g_{22}\right) \in \mathbb{R}^{4}\right.$ : $\left.g_{11} g_{22}=g_{12} g_{21}\right\}$ and observing a channel realization $\boldsymbol{g}$, such that, $g_{11} g_{22}=g_{12} g_{21}$ is a zero probability event, since all channel gains are drawn from continuous probability 
distributions. Thus, with probability one, the game $\mathcal{G}_{(a)}$ has unique NE. This completes the proof.

\section{Proof of Proposition 4}

In this appendix, we provide a proof of the Proposition 4 . The Proposition 4 basically states that at low SNR regime if an action profile $\boldsymbol{p}$ is a NE of the game $\mathcal{G}_{(a)}$, then it is also an NE of the game $\mathcal{G}_{(b)}$ and it is unique. The proof follows from the fact that in the asymptotic regime, i.e., SNR $\rightarrow 0$, the set $\mathcal{A}_{n}$ and the set $\mathcal{B}_{n}$ become identical, when $n \in\{1, \ldots, 4\}$. Moreover, the sets $\mathcal{A}_{m}$, with $m \in$ $\{5, \ldots 8\}$ become empty. The sets $\mathcal{A}_{1}, \ldots, \mathcal{A}_{4}$ and the sets $\mathcal{B}_{1}, \ldots, \mathcal{B}_{8}$ are given by Propositions 2 and 3 , respectively. Then,

$$
\begin{aligned}
\lim _{\mathrm{SNR} \rightarrow 0} \mathcal{A}_{1}=\lim _{\mathrm{SNR} \rightarrow 0} \mathcal{B}_{1}= & \left\{\boldsymbol{g} \in \mathbb{R}_{+}^{4}: \frac{g_{11}}{g_{12}} \geqslant 1\right. \text { and } \\
& \left.\frac{g_{21}}{g_{22}} \leqslant 1\right\}
\end{aligned}
$$

$$
\begin{aligned}
\lim _{\mathrm{SNR} \rightarrow 0} \mathcal{A}_{2}=\lim _{\mathrm{SNR} \rightarrow 0} \mathcal{B}_{2}= & \left\{\boldsymbol{g} \in \mathbb{R}_{+}^{4}: \frac{g_{11}}{g_{12}} \geqslant 1\right. \text { and } \\
& \left.\frac{g_{21}}{g_{22}} \geqslant 1\right\}
\end{aligned}
$$

$$
\begin{aligned}
\lim _{\mathrm{SNR} \rightarrow 0} \mathcal{A}_{3}=\lim _{\mathrm{SNR} \rightarrow 0} \mathcal{B}_{3}= & \left\{g \in \mathbb{R}_{+}^{4}: \frac{g_{11}}{g_{12}} \leqslant 1\right. \text { and } \\
& \left.\frac{g_{21}}{g_{22}} \leqslant 1\right\}
\end{aligned}
$$

$$
\begin{aligned}
\lim _{\mathrm{SNR} \rightarrow 0} \mathcal{A}_{4}=\lim _{\mathrm{SNR} \rightarrow 0} \mathcal{B}_{4}= & \left\{\boldsymbol{g} \in \mathbb{R}_{+}^{4}: \frac{g_{11}}{g_{12}} \leqslant 1\right. \text { and } \\
& \left.\frac{g_{21}}{g_{22}} \geqslant 1\right\}
\end{aligned}
$$

and moreover,

$$
\lim _{\mathrm{SNR} \rightarrow 0} \mathcal{A}_{5}=\left\{\boldsymbol{g} \in \mathbb{R}_{+}^{4}: \frac{g_{11}}{g_{12}}=1 \text { and } \frac{g_{21}}{g_{22}}=1\right\}
$$

and

$$
\forall n \in\{6, \ldots, 8\}, \quad \lim _{\mathrm{SNR} \rightarrow 0} \mathcal{A}_{n}=\emptyset .
$$

Now, since the sets $\mathcal{A}_{1}, \ldots, \mathcal{A}_{4}$ or the sets $\mathcal{B}_{1}, \ldots, \mathcal{B}_{4}$ cover, in the asymptotic regime, all the space of vectors $g$ and both $\mathcal{A}_{n}$ and $\mathcal{B}_{n}$ determine a unique NE in the game $\mathcal{G}_{(b)}$ and $\mathcal{G}_{(a)}$, respectively, it follows that the NE of both games is identical in the asymptotic regime. The uniqueness of the NE in the game $\mathcal{G}_{(a)}$ holds with probability one, independently of the SNR level (Proposition 2). In the game $\mathcal{G}_{(b)}$, the NE is not unique if and only if $\boldsymbol{g} \in \mathcal{A}_{5}$. Nonetheless, since for all $(k, s) \in \mathcal{K} \times \mathcal{S}, g_{k, s}$ is a realization of a random variable drawn from a continuous probability distribution, we have that

$$
\operatorname{Pr}\left(g \in \mathcal{A}_{5}\right)=0
$$

Thus, with probability one, the NE of the game $\mathcal{G}_{(b)}$ is unique in the low SNR regime, which completes the proof.

\section{Proof of Proposition 5}

In this appendix, we provide the proof of Proposition 5, which states that at the high SNR regime there always exists an NE action profile in the game $\mathcal{G}_{(b)}$, which leads to an equal or better global performance than the unique NE of the game $\mathcal{G}_{(a)}$. Before we start, we introduce two lemmas which are used in the proof.

Lemma 8. In the high $S N R$ regime, the game $\mathcal{G}_{(\text {a) }}$ possesses a unique NE, which can be of six different types depending on the channel realizations $\left\{g_{i j}\right\}_{\forall(i, j) \in \mathcal{K} \times \mathcal{S}}$ :

- Equilibrium 1: if $\boldsymbol{g} \in \mathcal{A}_{1}^{\prime}=\left\{\boldsymbol{g} \in \mathbb{R}_{+}^{4}: g_{22} \geqslant g_{12}\right.$, and $\left.g_{21} \leqslant g_{11}\right\}$, then, $p_{11}^{\dagger}=p_{\max }$ and $p_{22}^{\dagger}=p_{\max }$.

- Equilibrium 4: if $\boldsymbol{g} \in \mathcal{A}^{\prime}{ }_{4}\left\{\boldsymbol{g} \in \mathbb{R}_{+}^{4}: g_{11} \leqslant g_{21}\right.$, and $\left.g_{12} \geqslant g_{22}\right\}$, then, $p_{11}^{\dagger}=0$ and $p_{22}^{\dagger}=0$.

- Equilibrium 5: if $\boldsymbol{g} \in \mathcal{A}^{\prime}{ }_{5}=\left\{\boldsymbol{g} \in \mathbb{R}_{+}^{4}: \frac{g_{11}}{g_{12}} \geqslant \frac{g_{21}}{g_{22}}\right.$, and $\left.g_{21}>g_{11}\right\}$, then, $p_{11}^{\dagger}=p_{\max }$ and $p_{22}^{\dagger}=\frac{1}{2}\left(p_{\max }-\frac{\sigma^{2}}{g_{22}}+\frac{\sigma^{2}+g_{11} p_{\max }}{g_{21}}\right)$.

- Equilibrium 6: if $\boldsymbol{g} \in \mathcal{A}^{\prime}{ }_{6}=\left\{\boldsymbol{g} \in \mathbb{R}_{+}^{4}: \frac{g_{11}}{g_{12}} \geqslant \frac{g_{21}}{g_{22}}\right.$, and $\left.g_{22}<g_{12}\right\}$, then, $p_{11}^{\dagger}=\frac{1}{2}\left(p_{\max }-\frac{\sigma^{2}}{g_{11}}+\frac{\sigma^{2}+p_{\max } g_{22}}{g_{12}}\right)$ and $p_{22}^{\dagger}=p_{\max }$.

- Equilibrium 7: if $\boldsymbol{g} \in \mathcal{A}_{7}^{\prime}=\left\{\boldsymbol{g} \in \mathbb{R}_{+}^{4}: \frac{g_{11}}{g_{12}} \leqslant \frac{g_{21}}{g_{22}}\right.$, and $\left.g_{11}>g_{21}\right\}$, then, $p_{11}^{\dagger}=\frac{1}{2}\left(p_{\max }-\frac{\sigma^{2}+p_{\max } g_{21}}{g_{11}}+\frac{\sigma^{2}}{g_{12}}\right)$ and $p_{22}^{\dagger}=0$.

- Equilibrium 8: if $\boldsymbol{g} \in \mathcal{A}_{8}^{\prime}=\left\{\boldsymbol{g} \in \mathbb{R}_{+}^{4}: \frac{g_{11}}{g_{12}} \leqslant \frac{g_{21}}{g_{22}}\right.$, and $\left.g_{12}<g_{21}\right\}$, then, $p_{11}^{\dagger}=0$ and $p_{22}^{\dagger}=\frac{1}{2}\left(p_{\max }-\frac{\sigma^{2}+g_{12} p_{\max }}{g_{22}}+\frac{\sigma^{2}}{g_{21}}\right)$.

The proof of Lemma 8 follows the same reasoning of the proof of Proposition 2. Here, $\forall n \in\{1, \ldots, 8\}, \mathcal{A}_{n}^{\prime}=$ $\lim _{\mathrm{SNR} \rightarrow \infty} \mathcal{A}_{n}$, where the sets $\mathcal{A}_{1}, \ldots, \mathcal{A}_{8}$ are given by Proposition 2.

In the following lemma, we describe the set of NE of the game $\mathcal{G}_{(b)}$ in the high SNR regime.

Lemma 9. In the high SNR regime, the game $\mathcal{G}_{(b)}$ always possesses two NE action profiles:

$$
\boldsymbol{p}_{1}^{*, 1}=\left(0, p_{\max }\right) \text { and } \boldsymbol{p}_{2}^{*, 1}=\left(p_{\max }, 0\right)
$$

and

$$
\boldsymbol{p}_{1}^{*, 4}=\left(p_{\max }, 0\right) \text { and } \boldsymbol{p}_{2}^{*, 4}=\left(0, p_{\max }\right)
$$

independently of the channel realizations. 
In the high SNR, i.e., SNR $\rightarrow \infty$, the sets $\mathcal{B}_{1}, \ldots, \mathcal{B}_{4}$, given by Proposition 3, become the following sets,

$$
\begin{aligned}
& \lim _{\mathrm{SNR} \rightarrow+\infty} \mathcal{B}_{1}=\lim _{\mathrm{SNR} \rightarrow+\infty} \mathcal{B}_{4}=\mathbb{R}_{+}^{4} \\
& \lim _{\mathrm{SNR} \rightarrow+\infty} \mathcal{B}_{2}=\lim _{\mathrm{SNR} \rightarrow+\infty} \mathcal{B}_{3}=\emptyset .
\end{aligned}
$$

Thus, one can immediately imply that

$$
\operatorname{Pr}\left(g \in \lim _{\mathrm{SNR} \rightarrow+\infty} \mathcal{B}_{2}\right)=\operatorname{Pr}\left(g \in \lim _{\mathrm{SNR} \rightarrow+\infty} \mathcal{B}_{3}\right)=0
$$

and,

$$
\operatorname{Pr}\left(g \in \lim _{\mathrm{SNR} \rightarrow+\infty} \mathcal{B}_{1}\right)=\operatorname{Pr}\left(\boldsymbol{g} \in \lim _{\mathrm{SNR} \rightarrow+\infty} \mathcal{B}_{4}\right)=1
$$

Hence, from Proposition 3, we imply that both $\boldsymbol{p}^{(*, 1)}$ and $\boldsymbol{p}^{(*, 4)}$ are NE action profiles of the game $\mathcal{G}_{(b)}$ in the high SNR regime regardless of the exact channel realizations $\left\{g_{i j}\right\}_{\forall(i, j) \in \mathcal{K} \times \mathcal{S}}$, which completes the proof.

From Lemmas 8 and 9 , it is easy to see that if $\boldsymbol{g}=$ $\left(g_{11}, g_{12}, g_{21}, g_{22}\right) \in \mathcal{A}_{n}^{\prime}$, with $n \in\{1,4\}$, then (21) holds since $\boldsymbol{p}^{\dagger}$ and at least one of the NE action profiles $\boldsymbol{p}^{*, n}$, with $n \in\{1,4\}$ are identical. In the cases where $g=$ $\left(g_{11}, g_{12}, g_{21}, g_{22}\right) \in \mathcal{A}_{n}^{\prime}$, with $n \in\{5, \ldots, 8\}$, we prove by inspection that in all the cases condition (21) always holds for both NE action profiles $\boldsymbol{p}^{(*, 1)}$ and $\boldsymbol{p}^{(*, 4)}$. For instance, assume that $\boldsymbol{g} \in \mathcal{A}^{\prime}{ }_{5}$. Then, we have that the unique NE of the game $\mathcal{G}_{(a)}$ is $\boldsymbol{p}^{\dagger}=\left(p_{11}^{\dagger}, p_{\max }-p_{11}^{\dagger}, p_{\max }-p_{22}^{\dagger}, p_{22}^{\dagger}\right)$, with $p_{11}^{\dagger}=p_{\max }$ and $p_{22}^{\dagger}=\frac{1}{2}\left(p_{\max }+\frac{\sigma^{2}+p_{\max } g_{11}}{g_{21}}-\frac{\sigma^{2}}{g_{22}}\right)$ (see Lemma 8). Define the function $\psi: \mathbb{R}_{+} \rightarrow \mathbb{R}_{+}$as follows: $\psi(x)=1+\mathrm{SNR} x$, with SNR $=\frac{p_{\max }}{\sigma^{2}}$, and denote by $\Delta_{1}$ (SNR), the difference between the NSE achieved by playing $\mathcal{G}_{(a)}$ and $\mathcal{G}_{(b)}$, with respect to the NE $\boldsymbol{p}^{*, 1}$ at SNR level SNR, i.e.,

$$
\begin{aligned}
\Delta_{1}(\mathrm{SNR})= & u_{1}\left(\boldsymbol{p}^{*, 1}\right)+u_{2}\left(\boldsymbol{p}^{*, 1}\right)- \\
& \left(u_{1}\left(\boldsymbol{p}^{\dagger}\right)+u_{2}\left(\boldsymbol{p}^{\dagger}\right)\right) \\
= & 2 \log _{2}(2)-2 \log _{2}\left(1+\frac{g_{21}}{g_{22}} \frac{\psi\left(g_{22}\right)}{\psi\left(g_{11}\right)}\right)- \\
& \log _{2}\left(1+\frac{g_{22}}{g_{21}} \frac{\psi\left(g_{11}\right)}{\psi\left(g_{22}\right)}\right)+ \\
& \log _{2}\left(\frac{g_{21}}{g_{22}}+\psi\left(g_{21}-g_{11}\right)\right) .
\end{aligned}
$$

Note that if $\boldsymbol{g} \in \mathcal{A}_{5}^{\prime}$, then $g_{21}>g_{11}$. Hence,

$$
\lim _{\mathrm{SNR} \rightarrow \infty} \Delta_{1}(\mathrm{SNR})=\infty
$$

which justifies (21). Similarly, denote $\Delta_{4}$ (SNR), the difference between the NSE achieved by playing $\mathcal{G}_{(a)}$ and $\mathcal{G}_{(b)}$, with respect to the $\mathrm{NE} \boldsymbol{p}^{*, 4}$, i.e.,

$$
\begin{aligned}
\Delta_{4}(\mathrm{SNR})= & u_{1}\left(\boldsymbol{p}^{*, 4}\right)+u_{2}\left(\boldsymbol{p}^{*, 4}\right)- \\
& \left(u_{1}\left(\boldsymbol{p}^{\dagger}\right)+u_{2}\left(\boldsymbol{p}^{\dagger}\right)\right) \\
= & 2 \log _{2}(2)-2 \log _{2}\left(1+\frac{g_{22}}{g_{21}} \frac{\psi\left(g_{21}\right)}{\psi\left(g_{12}\right)}\right) \\
& -\log _{2}\left(1+\frac{g_{21}}{g_{22}} \frac{\psi\left(g_{12}\right)}{\psi\left(g_{21}\right)}\right) \\
& +\log _{2}\left(\frac{g_{22}}{g_{21}}+\psi\left(g_{22}-g_{12}\right)\right) .
\end{aligned}
$$

Note that if $\boldsymbol{g} \in \mathcal{A}^{\prime}$, then $g_{22}>g_{12}$. Hence,

$$
\lim _{\mathrm{SNR} \rightarrow \infty} \Delta_{4}(\mathrm{SNR})=\infty,
$$

which justifies (21). Hence, one can imply that in the high SNR regime both NE action profiles $\boldsymbol{p}^{*, 1}$ and $\boldsymbol{p}^{*, 2}$, satisfy (21) when $\boldsymbol{g} \in \mathcal{A}^{\prime}{ }_{5}$. The same result as the one obtained when $\boldsymbol{g} \in \mathcal{A}_{5}^{\prime}$, is also obtained when $\boldsymbol{g} \in \mathcal{A}_{n}^{\prime}$, with $n \in$ $\{6, \ldots, 8\}$, which completes the proof.

\section{Endnote}

${ }^{\text {a }}$ Note that the PoS as defined in (29) is the multiplicative inverse of the one defined in [38].

\section{Competing interests}

The authors declare that they have no competing interests.

\section{Author details}

${ }^{1}$ Department of Electrical Engineering, Princeton University, Equad, 19 Olden Street, Princeton, NJ 08544, USA. ${ }^{2}$ Alcatel-Lucent Chair in Flexible Radio at SUPELEC, 3 rue Joliot-Curie, 91192, Gif-sur-Yvette, Cedex, France. ${ }^{3}$ LSS (CNRS SUPELEC - University Paris-Sud), 3 rue Joliot-Curie, 91192, Gif-sur-Yvette,

Cedex, France.

Received: 8 December 2011 Accepted: 15 November 2012

Published: 25 January 2013

\section{References}

1. TM Cover, JA Thomas, Elements of Information Theory (Wiley-Interscience, Hoboken, NJ, USA, 1991)

2. S Chieochan, E Hossain, J Diamond, Channel assignment schemes for infrastructure-based 802.11 WLANs: a survey. IEEE. Commun. Surv. Tutor. 12,1115-1129 (2010)

3. S Lasaulce, A Suarez, R de Lacerda, M Debbah, Using cross-system diversity in heterogeneous networks: throughput optimization. Performance Eval. 65(11), 907-921 (2008)

4. SM Perlaza, EV Belmega, S Lasaulce, M Debbah, in Proceedings of the 3rd ACM-ICST/International Workshop on Game Theory in Communications Networks (GAMECOMM). On the base station selection and base station sharing in self-configuring networks (Pisa, Italy, 2009)

5. F Meshkati, M Chiang, HV Poor, SC Schwartz, A game-theoretic approach to energy-efficient power control in multi-carrier CDMA systems. IEEE. J. Sel. Areas. Commun. 24(6), 1115-1129 (2006)

6. JF Nash, Equilibrium points in N-person games. Proc. Natl. Acad. Sci. USA. 36, 48-49 (1950)

7. L Rose, SM Perlaza, S Lasaulce, M Debbah, Learning equilibria with partial information in wireless networks. IEEE. Commun. Mag. (Special Issue Game Theory in Wireless Communications). 49(8), 136-142 (2011) 
8. S Lasaulce, H Tembine, Game Theory and Learning in Wireless Networks: Fundamentals and Applications. (Elsevier Academic Press, Waltham, MA, USA, 2011)

9. P Mertikopoulos, EV Belmega, AL Moustakas, S Lasaulce, Distributed learning policies for power allocation in multiple access channels. IEEE J. Sel. Areas Commun. 30, 96-106 (2012)

10. EV Belmega, S Lasaulce, M Debbah, Power allocation games for MIMO multiple access channels with coordination. IEEE. Trans. Wirel. Commun. 8(6), 3182-3192 (2009)

11. L Lai, $\mathrm{H}$ El, Gamal, The water-filling game in fading multiple-access channels. IEEE. Trans. Inf. Theory. 54(5), 2110-2122 (2008)

12. G He, M Debbah, E Altman, A Bayesian game-theoretic approach for distributed resource allocation in fading multiple access channels. EURASIP J. Wirel. Commun. Netw. 2010, 1-12 (2010)

13. W Yu, W Rhee, S Boyd, J Cioffi, Iterative water-filling for Gaussian vector multiple-access channels. IEEE. Trans. Inf. Theory. 50, 145-152 (2004)

14. G Scutari, DP Palomar, S Barbarossa, The MIMO iterative waterfilling algorithm. IEEE. Trans. Signal Process. 57(5), 1917-1935 (2009)

15. RA Berry, DNC Tse, Shannon meets Nash on the interference channel. IEEE. Trans. Inf. Theory. 57(5), 2821-2836 (2011)

16. EG Larsson, EA Jorswieck, J Lindblom, R Mochaourab, Game theory and the flat-fading Gaussian interference channel: analyzing resource conflicts in wireless networks. IEEE. Signal. Process Mag. (Special issue on Game Theory for Signal Processing). 26(5), 18-27 (2009)

17. EG Larsson, E Jorswieck, Competition versus cooperation on the MISO interference channel. IEEE J. Sel. Areas Commun. 26(7), 1059-1069 (2008)

18. W Yu, J Cioffi, Constant-power waterfilling: performance bound and low-complexity implementation. IEEE. Trans. Commun. 54, 23-28 (2006)

19. G Scutari, DP Palomar, S Barbarossa, Optimal linear precoding strategies for wideband noncooperative systems based on game theory-Part I: Nash equilibria. IEEE. Trans. Signal Process. 56(3), 1230-1249 (2008)

20. L Rose, SM Perlaza, M Debbah, in Proceedings of the International IEEE Conference on Communications (ICC). On the Nash equilibria in decentralized parallel interference channels (Kyoto, Japan, 2011)

21. D Monderer, LS Shapley, Potential games. Games. Econ. Behav. 14, 124-143 (1996)

22. M Voorneveld, Best-response potential games. Econ. Lett. 66(3), 289-295 (2000)

23. D Braess, Über ein Paradoxon aus der Verkehrsplanung. Unternehmensforschung. 24(5), 258-268 (1969)

24. E Altman, T Jimenez, N Vicuna, R Marquez, in Proceedings of the 6 th International Symposium on Modeling and Optimization in Mobile, Ad Hoc, and Wireless Networks (WiOPT). Coordination games over collision channels, (Berlin, Germany, 2008)

25. E Altman, $\mathrm{V}$ Kumar, $\mathrm{H}$ Kameda, in Proceedings of the 6th International Symposium on Modeling and Optimization in Mobile, Ad Hoc, and Wireless Networks (WiOPT). A Braess type paradox in power control over interference channels (Berlin, Germany, 2008)

26. E Telatar, Capacity of multi-antenna Gaussian channels. Eur. Trans Telecommun. 10(6), 585-596 (1999)

27. E Telatar, Capacity of multi-antenna Gaussian channels. Technical report, Lucent Technologies, Bell Laboratories, Murray Hill, NJ, USA (1995)

28. G Scutari, S Barbarossa, DP Palomar, in Proceedings of the International Conference on Acoustics, Speech and Signal Processing (ICASSP). Potential games: a framework for vector power control problems with coupled constraints, (Toulouse, France, 2006)

29. SM Perlaza, H Tembine, S Lasaulce, VQ Florez, in Proceedings of the IEEE Latin-American Conference on Communications (LATINCOM). On the fictitious play and channel selection games (Bogotá, Colombia, 2010)

30. G Scutari, DP Palomar, S Barbarossa, Optimal linear precoding strategies for wideband non-cooperative systems based on game theory-Part II: algorithms. IEEE. Trans. Signal Process. 56(3), 1250-1267 (2008)

31. K Fan, Fixed-point and minimax theorems in locally convex topological linear spaces. Proc. Natl. Acad. Sci. USA. 38(2), 121-126 (1952)

32. SM Perlaza, Game theoretic approaches to spectrum sharing in decentralized self-configuring networks. PhD thesis, Télécom ParisTech (2011)

33. T Ui, Discrete concavity for potential games. Int. Game Theory Rev 10(01), 137-143 (2008)

34. E Altman, RE Azouzi, O Pourtallier, Avoiding paradoxes in multi-agent competitive routing. Comput. Netw. 43(2), 133-146 (2003)
35. SM Perlaza, M Debbah, S Lasaulce, H Bogucka, in Proceedings of the IEEE International Conference on Cognitive Radio Oriented Wireless Networks and Communications (CROWNCOM). On the benefits of bandwidth limiting in decentralized vector multiple access channels (Hannover, Germany, 2009)

36. E Belmega, S Lasaulce, M Debbah, in Proceedings of the 3rd International Symposium on Communications, Control and Signal Processing (ISCCSP). Decentralized handovers in cellular networks with cognitive terminals (St. Julians, Malta, 2008)

37. E Koutsoupias, C Papadimitriou, in Proceedings of the 16th Annual Symposium on Theoretical Aspects of Computer Science. Worst-case equilibria, (Trier, Germany, 1999)

38. E Anshelevich, A Dasgupta, E Tardos, in Proceedings of the Annual ACM Symposium on Theory of Computing. Near-optimal network design with self-fish agents (San Diego, CA, USA, 2003)

doi:10.1186/1687-1499-2013-15

Cite this article as: Perlaza et al.: Equilibria of channel selection games in parallel multiple access channels. EURASIP Journal on Wireless Communications and Networking 2013 2013:15.

\section{Submit your manuscript to a SpringerOpen ${ }^{\circ}$ journal and benefit from:}

- Convenient online submission

Rigorous peer review

- Immediate publication on acceptance

- Open access: articles freely available online

- High visibility within the field

- Retaining the copyright to your article

Submit your next manuscript at $>$ springeropen.com 